Research Article

\title{
Multiobjective Design Optimization Framework for Multicomponent System with Complex Nonuniform Loading
}

\author{
Hong Zhang, Guangchen Bai, and Lukai Song iD \\ School of Energy and Power Engineering, Beihang University, Beijing 100191, China \\ Correspondence should be addressed to Lukai Song; songlukai29@163.com
}

Received 3 July 2020; Accepted 30 July 2020; Published 19 August 2020

Guest Editor: Shun-Peng Zhu

Copyright ( 2020 Hong Zhang et al. This is an open access article distributed under the Creative Commons Attribution License, which permits unrestricted use, distribution, and reproduction in any medium, provided the original work is properly cited.

To improve the accuracy and efficiency of multiobjective design optimization for a multicomponent system with complex nonuniform loads, an efficient surrogate model (the decomposed collaborative optimized Kriging model, DCOKM) and an accurate optimal algorithm (the dynamic multiobjective genetic algorithm, DMOGA) are presented in this study. Furthermore, by combining DCOKM and DMOGA, the corresponding multiobjective design optimization framework for the multicomponent system is developed. The multiobjective optimization design of the carrier roller system is considered as a study case to verify the developed approach with respect to multidirectional nonuniform loads. We find that the total standard deviation of three carrier rollers is reduced by $92 \%$, where the loading distribution is more uniform after optimization. This study then compares surrogate models (response surface model, Kriging model, OKM, and DCOKM) and optimal algorithms (neighbourhood cultivation genetic algorithm, nondominated sorting genetic algorithm, archive microgenetic algorithm, and DMOGA). The comparison results demonstrate that the proposed multiobjective design optimization framework is demonstrated to hold advantages in efficiency and accuracy for multiobjective optimization.

\section{Introduction}

Multicomponent system is defined as the complex mechanism system comprising a plurality of rigid and flexible components, which is an indispensable part in mechanical equipment, such as excavator and loader [1-3]. For example, the carrier roller system of the excavator is a typical multicomponent system. Such multicomponent system, however, suffers from nonuniform loads caused by complex structural layout and stricter working environment, and the failure of one component will lead to the failure of the whole component system, which significantly increases the failure possibility of the multicomponent system. In addition, the multicomponent system tends to have the mutual conduction effect between components, and the design and control of one structural objective often leads to the change of another. Therefore, to reduce the load nonuniformity and improve the system performance, it is necessary to perform the multiobjective design optimization (MODO) for the complex multicomponent system. A typical MODO problem can be formulated as
Find $x$

$$
\begin{array}{r}
\min \sigma\left\{f_{i}(x)\right\} \\
\text { S.t. }\left\{\begin{array}{l}
\sum F_{j}<\sum\left[F_{j}\right] \\
g_{l}(x)=0 \\
a_{u} \leq x_{u} \leq b_{u},
\end{array}\right.
\end{array}
$$

where $f_{i}(x)$ denotes the $i$-th objective function; $F_{j}$ is the $j$-th constraint function; $\left[F_{j}\right]$ is the allowable load under $j$-th constraint function; $g_{l}(x)$ is the equality constraint of the $l$ th design parameter $x ; x_{u}$ is the $u$-th design parameter; and $a_{u}$ and $b_{u}$ are the upper and bottom boundaries of the parameter $x$, respectively; $i=1,2, \ldots, m ; j=1,2, \ldots, n ; u=1$, $2, \ldots, p ; l=1,2, \ldots, q$.

To solve the MODO model shown in (1), the multiple response simulations (i.e., objective response simulation and constraint response simulation) and multiobjective optimal algorithm are unavoidable to be required. Under such circumstances, it will require thousands of calls to expensive 
black-box function to simulate the multiobjective and multiconstraint, which will lead to huge computing task and unaffordable computing cost. To improve the computational efficiency, differentiation algorithms, generalized speeds, Euler parameters, lower body arrays, and other dynamic numerical methods are proposed and widely applied [4-6]. Unfortunately, although these numerical methods can greatly reduce simulation time, the approximation accuracy of multiobjective and multiconstraint is unacceptable for the complex multicomponent system. Moreover, the nonlinear constrain space and multiple optimal solutions usually demand nested iterative loops, which also brings the solving difficulties of the MODO model. To improve MODO accuracy and efficiency of the complex multicomponent system, this study presents two key techniques: (1) establishment of a numerical surrogate model to calculate the multiobjective and multiconstraint; (2) development of a dynamic multiobjective algorithm to resolve the MODO model.

The first technique is to establish an efficient surrogate model to approximate the multiobjective and multiconstraint. Only needing a small amount of black-box function calls, the surrogate model can be established and is promising to reduce simulation cost [7-11]. Classic surrogate models include polynomial response surface [12-14], Kriging model (KM) [15-17], artificial neural network [18-20], and support vector regression [21-23]. Among them, with integrating global nonlinear approximation ability and local precise description ability, the Kriging model possesses the potentials to approximate complex structural responses and ensure calculation accuracy $[24,25]$, which is suitable for complex multiobjective optimization problems of the multicomponent system. However, with regard to high-nonlinearity traits of multiobjective and multiconstraint problems, the traditional Kriging model is still insufficient to acquire satisfactory efficiency and accuracy. To address this problem, in this study, by utilizing an improved particle swarm optimization algorithm [26] to search Kriging key parameters and decomposed collaborative strategy [27-30] to simplify calculation complexity, a decomposed collaborative optimized Kriging model (DCOKM) is proposed to complete efficient approximation of multiobjective and multiconstraint.

The second technique is to develop an accurate multiobjective optimal algorithm to resolve the complex MODO model. Due to the complex interaction effects between various objective functions in the multicomponent system, the global optimal solution of the MODO model is almost impossible to acquire at the same time. Therefore, an accurate optimal algorithm, which can precisely search for Pareto optimal frontier and acquire nondominated solutions, is urgently required. At present, a variety of multiobjective optimization algorithms are developed, such as multi-island genetic algorithm (MIGA) [31-33], neighbourhood cultivation genetic algorithm (NCGA) [34, 35], the second generation of nondominated sorting genetic algorithm (NSGA-II) [36-38], and archive microgenetic algorithm (AMGA)
[39, 40]. Among them, owing to the virtues of nondominated sorting strategy and elitist retention strategy, NSGA-II has been widely used in MODO problems. Unfortunately, because of easy to fall into local solutions or premature convergence, the current NSGA-II algorithm is still difficult to acquire global optimal solution of the multicomponent system [41, 42]. In this case, by designing an arithmetic crossover operator and Poisson mutation operator in NSGA-II algorithm, a dynamic multiobjective genetic algorithm (DMOGA) is presented to accurately solve the MODO model and acquire nondominated solutions.

The objective of this paper is to develop an efficient and accurate multiobjective design optimization framework for the multicomponent system with nonuniform loads. By fusing the proposed surrogate model (i.e., DCOKM) and optimal algorithm (i.e., DMOGA), the urgently needed multiobjective design optimization framework is established. The innovation of this paper lies in that the corresponding efficient methods are firstly proposed for the multiobjective optimization design of the multicomponent system with nonuniform loading. Regarding the multiobjective design optimization of the carrier roller system as study case, the effectiveness of the presented framework is validated. In what follows, Section 2 investigates surrogate modeling with DCOKM, optimal algorithm with DMOGA, and the corresponding multiobjective design optimization framework. Section 3 performs the study case to validate the multiobjective design optimization framework. Some conclusions are summarized in Section 4.

\section{Basic Theories and Formulations}

\subsection{Decomposed Collaborative Optimized Kriging Model, DCOKM}

2.1.1. Optimized Kriging Model, OKM. By integrating the Kriging model (KM) with the best unbiasedness ability and the improved particle swarm optimization (IPSO) with global search capability [43], the optimized Kriging model (OKM) is introduced as

$$
y(x)=f^{T}(x) \cdot \beta+z(x)
$$

where $x=\left[x_{1}, x_{2}, \ldots, x_{d}\right]$ indicates the input variable; $d$ is the dimension number of input parameters; $f^{T}(x) \beta$ signifies the global approximation trend; $f^{T}(x)=\left[f_{1}(x), f_{2}(x), \ldots, f_{m}(x)\right]$ is the regression basis function; $\beta=\left[\beta_{1}, \beta_{2}, \ldots, \beta_{m}\right]$ is the regression coefficient; $m$ is the dimension number of regression function; and $z(x)$ is the local random deviation, which follows Gaussian distribution $\left(0, \sigma^{2}\right)$. Herein, the covariance measure of $z(x)$ is defined as

$$
\operatorname{Cov}\left[z\left(x_{i}\right), z\left(x_{i}\right)\right]=\sigma^{2} R\left(x_{i}, x_{j}\right)
$$

where $x_{i}$ and $x_{j}$ denote the $i$-th and the $j$-th input variables, respectively; $R(\cdot, \cdot)$ is the Gauss correlation function, which is 


$$
\begin{aligned}
R\left(x_{i}, x_{j}\right) & =\exp \left(-\sum_{k=1}^{n} \theta_{k}\left|x_{i}^{(k)}-x_{j}^{(k)}\right|^{2}\right) \\
& =\prod_{k=1}^{n} \exp \left(-\theta_{k}\left|x_{i}^{(k)}-x_{j}^{(k)}\right|^{2}\right)
\end{aligned}
$$

in which $n$ represents the dimension number of design variables; $x(k) i$ and $x(k) j$ are the $k$-th member of variables $x_{i}$ and $x_{j}$, respectively; $\theta_{k}$ is the $k$-th member of correlation parameter $\theta$.

Moreover, the optimal correlation parameter $\theta^{*}$ of OKM can be obtained by the following maximum likelihood estimation, i.e.,

$$
\boldsymbol{\theta}^{*}=\operatorname{argmax}\{\psi(\theta)\}=\arg \min \left\{\frac{1}{2}\left(\ln |\mathbf{R}|+m \ln \widehat{\sigma}^{2}\right)\right\},
$$

where $\psi(\theta)$ indicates the likelihood function.

Then, the corresponding regression coefficient $\beta$ and variance $\sigma^{2}$ of OKM can be estimated by

$$
\left\{\begin{array}{l}
\widehat{\boldsymbol{\beta}}=\left(\mathbf{F}^{T} \mathbf{R}^{-1} \mathbf{F}\right)^{-1} \mathbf{F}^{T} \mathbf{R}^{-1} \mathbf{y}, \\
\widehat{\sigma}^{2}=\frac{1}{n}(\mathbf{y}-\mathbf{F} \widehat{\boldsymbol{\beta}})^{T} \mathbf{R}^{-1}(\mathbf{y}-\mathbf{F} \widehat{\boldsymbol{\beta}}),
\end{array}\right.
$$

where $\boldsymbol{F}$ is the unit column vector and $\boldsymbol{R}$ is the correlation matrix.

Clearly, the approximation accuracy of OKM mostly depends on the correlation parameter $\boldsymbol{\theta}$. Once the optimal correlation parameter $\boldsymbol{\theta}^{*}$ is acquired, the optimal unbiased prediction of OKM is ensured. Therefore, the high-accurate surrogate modeling problem is transformed into the searching problem of the optimal solution $\boldsymbol{\theta}^{*}$.

Searching algorithm is a key factor to find the optimal solution $\boldsymbol{\theta}^{*}$, since its searching efficiency and accuracy directly influence the approximation efficacy of the Kriging model. The PSO algorithm is an important nongradient searching algorithm, and it holds good collaborative searching ability [26]. However, the fixed inertia weight and learning factors of the ordinary PSO algorithm often fall into blind searching and trap by local optimum easily, which greatly affects the searching efficiency and accuracy. To address this issue, we propose an improved PSO algorithm by designing adaptive inertia weight and asynchronous learning factors, with which the iteration steps change. The design is to acquire better optimal solution $\boldsymbol{\theta}^{*}$ by balancing the global searching ability and local searching ability. The basic thought of improved PSO algorithm is summarized as follows.

We assume that the particle position is assigned as the correlation parameter $\boldsymbol{\theta}$ and the fitness value adopts the $\psi(\theta)$ of the optimized Kriging model. Hereinto, each particle is a potential solution for the optimal correlation parameter $\boldsymbol{\theta}^{*}$. In searching process, with following the direction guidance of optimal particles, the particle swarm will search for the optimal solution by updating particle individual positions, individual extreme values, and population extremum values. The renewal formulas of the improved PSO algorithm are introduced as

$$
\begin{array}{r}
\left\{\begin{array}{l}
\mathbf{V}_{i}^{k+1}=\omega \mathbf{V}_{i}^{k}+c_{1} r_{1}\left(\mathbf{P}_{i}^{k}-\mathbf{X}_{i}^{k}\right)+c_{2} r_{2}\left(\mathbf{P}_{g}^{k}-\mathbf{X}_{i}^{k}\right) \\
\mathbf{X}_{i}^{k+1}=\mathbf{X}_{i}^{k}+\mathbf{V}_{i}^{k+1}
\end{array} \quad(i=1,2, \cdots, N),\right.
\end{array}
$$

in which the adaptive inertia weight $\omega$ and asynchronous learning factors $c$ are

$$
\begin{aligned}
& \omega= \begin{cases}\omega_{\max }-\left(\omega_{\max }-\omega_{\min }\right) \frac{f-f_{\min }}{f_{\text {avg }}-f_{\min }}, & f \leq f_{\text {avg }}, \\
\omega_{\max }, & f>f_{\text {avg }},\end{cases} \\
& c=\left\{\begin{array}{l}
c_{1}=c_{1 s}+\left(c_{1 n}-c_{1 s}\right) \times(k / K), \\
c_{2}=c_{2 s}+\left(c_{2 n}-c_{2 s}\right) \times(k / K),
\end{array}\right.
\end{aligned}
$$

where $i$ represents the $i$-th particle; $k$ is the current iteration number; $K$ is the largest iteration number; $\omega$ is the inertia weight; $V_{i}$ is the current particle velocity; $\mathbf{X}_{i}$ is the current particle position; $\mathbf{P}_{i}$ is the current individual extremum; $\mathbf{P}_{g}$ is the current population extremum; $c_{1}$ and $c_{2}$ are the individual and population leaning factors, respectively; $r_{1}$ and $r_{2}$ are the random numbers during time domain $[0,1] ; \omega_{\max }$ is the maximum inertia weight; $\omega_{\min }$ is the minimum inertia weight; $f$ is the current fitness value; $f_{\text {avg }}$ is the average fitness value; $f_{\min }$ is the minimum fitness value; $c_{1 s}$ and $c_{2 s}$ are the initial individual learning factor and initial population learning factor, respectively; and $c_{1 n}$ and $c_{2 n}$ are the individual learning factor and population learning factor in the largest iterations, respectively.

With finite iteration times of the improved PSO algorithm, the specified searching precision is met and the optimal correlation parameters $\boldsymbol{\theta}^{*}$ are acquired. In light of Kriging interpolation theory, the best unbiased prediction of OKM is achieved, and the predicted response $\hat{y}(\tilde{x})$ related to the predicted point $\tilde{x}$ outside sample set can be expressed by

$$
\hat{y}(\widetilde{\mathbf{x}})=\mathbf{f}^{T}(\widetilde{\mathbf{x}}) \widehat{\boldsymbol{\beta}}+\mathbf{r}^{T}(\widetilde{\mathbf{x}}) \mathbf{R}^{-1}(\mathbf{y}-\mathbf{F} \widehat{\boldsymbol{\beta}}),
$$

where $\mathbf{r}=\left[R\left(\widetilde{x}, x_{1}\right), R\left(\widetilde{x}, x_{2}\right), \ldots, R\left(\widetilde{x}, x_{n}\right)\right]^{\mathrm{T}}$ is the correlation vector between an predicted point $\widetilde{\mathbf{x}}$ and training sample points $\left(x_{1}, x_{2}, \ldots, x_{n}\right)$.

With the predicted response $\widehat{y}(\tilde{x})$, the prediction error of OKM can be evaluated by

$$
\begin{aligned}
\varphi(\widetilde{\mathbf{x}}) & =\widehat{\sigma}^{2}\left[1+\mathbf{u}^{T}(\widetilde{\mathbf{x}})\left(\mathbf{F}^{T} \mathbf{R}^{-1} \mathbf{F}\right)^{-1} u(\widetilde{\mathbf{x}})-r^{T}(\widetilde{\mathbf{x}}) \mathbf{R}^{-1} r(\widetilde{\mathbf{x}})\right] \\
\text { s.t. } u(\widetilde{\mathbf{x}}) & =\mathbf{F}^{T} \mathbf{R}^{-1} r(\widetilde{\mathbf{x}})-f(\widetilde{\mathbf{x}}) .
\end{aligned}
$$

Noticeably, by combining the nonlinear approximation ability of KM and the powerful global searching ability of the improved PSO algorithm, the presented OKM holds the 

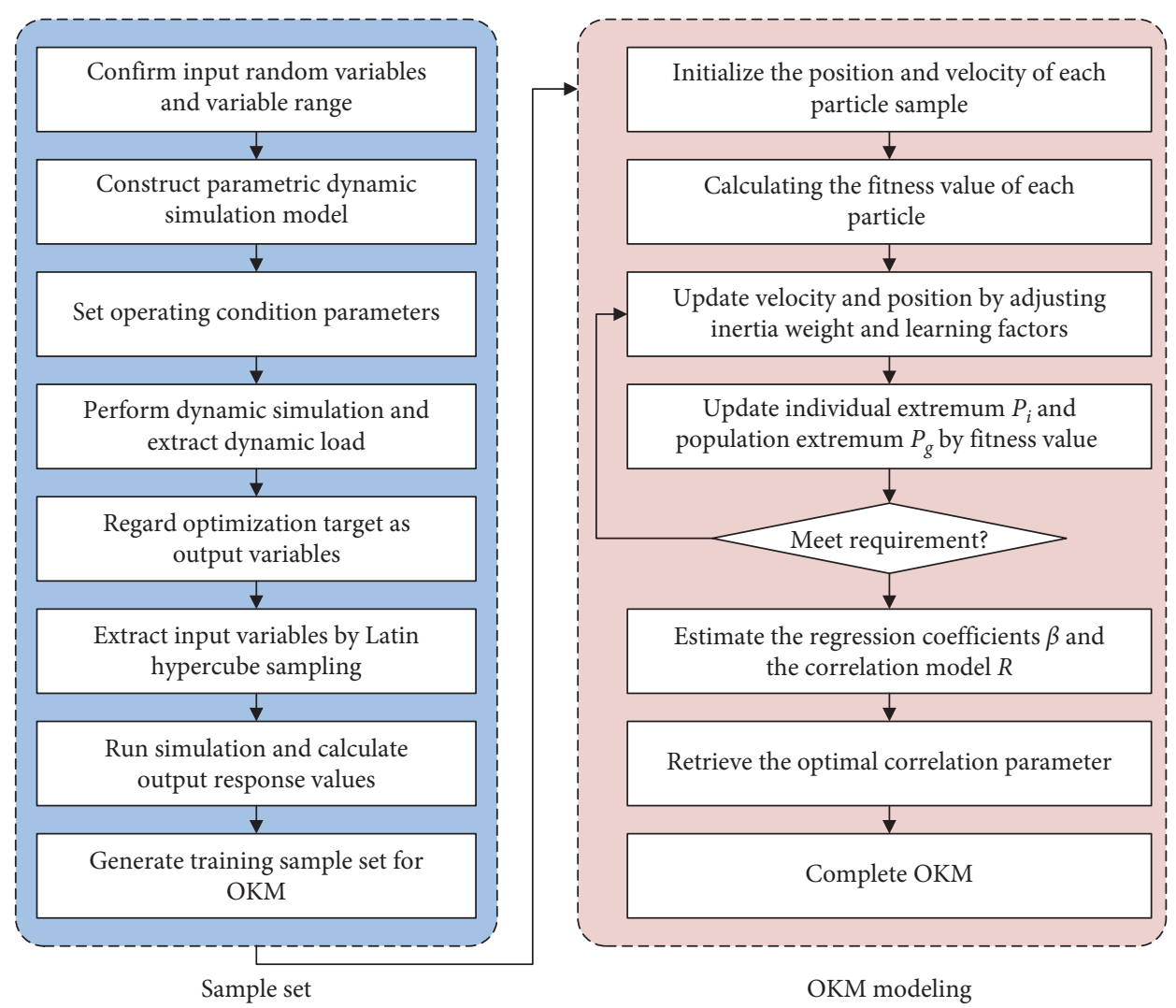

Figure 1: Flowchart of OKM. The establishment process of the OKM model is described.

potential to improve approximate efficiency and accuracy. The flowchart of OKM is drawn in Figure 1.

2.1.2. Mathematical Modeling of DCOKM. Multiobjective design optimization of the multicomponent system refers to multiobjective (i.e., the force standard deviation in the first component $\sigma_{F 1}$, in the third component $\sigma_{F 3}$, and in all rollers $\sigma_{M F}$, etc.) and multiconstraint (i.e., the sum of mean force in all rollers $S_{M F}$ and the sum of maximum force in all rollers $S_{F \max }$, etc.), which is difficult to perform the nonlinear approximation with high efficiency and acceptable accuracy. To address the multiobjective and multiconstraint (MOMC) problem, we develop a decomposed collaborative optimized Kriging model (DCOKM) with respect to the decomposed collaborative strategy and optimized Kriging model, to further address the strong nonlinearity issues and enhance approximation efficacy. For the MOMC approximation with the presented DCOKM, an entire complex MOMC system with all input variables and output responses is decomposed into many single-objective single-constraint subsystems, in which each submodel has few input variables and output responses. Considering Latin hypercube sampling technique and OKM, the decomposed OKM for many single-objective single-constraints is established. Subsequently, considering collaborative sampling technique and decomposed OKM, the DCOKM is mathematically constructed. The mathematical modeling process of DCOKM is summarized.
Assuming that the multiobjective and multiconstraint problem involves $r$ objectives and $l$ constraints, when $\boldsymbol{x}^{(t)}$ indicates the $t$-th input sample, then the corresponding $t$-th output response $Y^{(t)}$ is

$$
\left\{\begin{array}{l}
\mathbf{Y}^{(t)}=f\left(\mathbf{x}^{(t)}\right), \\
\mathbf{x}^{(t)}=\left[x_{1}^{(t)}, x_{2}^{(t)}, \ldots, x_{n}^{(t)}\right]^{T}, \\
t=1,2, \ldots, p, p+1, \ldots, l,
\end{array}\right.
$$

where $t=1,2, \ldots, p$ indicates output objective, while $t=p+1, p+2, \ldots, l$ represents the constraint response.

With a given predicted point $\widetilde{\mathbf{x}}^{(t)}$ for (10), the corresponding output response $\widetilde{\mathbf{Y}}^{(t)}$ is

$$
\widetilde{\mathbf{Y}}^{(t)}=\mathbf{f}^{T}\left(\widetilde{\mathbf{x}}^{(t)}\right) \widehat{\boldsymbol{\beta}}^{(t)}+\mathbf{r}^{T}\left(\widetilde{\mathbf{x}}^{(t)}\right)\left[\mathbf{R}^{(t)}\right]^{-1}\left(\mathbf{y}^{(t)}-\mathbf{F} \widehat{\boldsymbol{\beta}}^{(t)}\right) .
$$

Equation (13) is the decomposed OKM under $p$-th objective response and $l$-th constraint response, in which $\widehat{\beta}^{(t)}$, $R^{(t)}$, and $r^{\mathrm{T}}\left(\widetilde{\mathbf{x}}^{(t)}\right)$ indicate the optimal regression coefficient, the correlation model, and the correlation vector in decomposed OKM, respectively. From the above analysis, the OKM (10) of multiobjective and multiconstraint models is divided into a series of OKM submodels under single-objective and single-constraint such as (12) and (13), and the approximation complexity is significantly reduced. As the efficient and accurate DCOKM is developed, the MOMC problem is promising to be addressed for multiobjective design optimization. 
2.2. Dynamic Multiobjective Genetic Algorithm, DMOGA. The resolving algorithm is a critical factor for solving the MODO model and obtaining the optimal solutions. NSGAII is a vital nondominated fast sorting algorithm in multiobjective optimal design, which can effectively control the population distribution and reduce the resolving complexity. However, due to randomness and uncertain fluctuation effects in simulated binary crossover and polynomial mutation operator, its convergence precision is still unacceptable for the complex MODO model. To tackle with this problem, by designing the arithmetic crossover operator and Poisson mutation operator, we propose a dynamic multiobjective genetic algorithm (DMOGA) based on the traditional NSGA-II. The objective of the DMOGA is to reduce the uncertain fluctuation effects of operators and gain ideal Pareto optimal frontier. The design thought of the arithmetic crossover operator and Poisson mutation operator is presented as follows.

To improve the population diversity and avoid over propagation of excellent solutions, an arithmetic crossover operator is presented by combining the information of nondominated sorting levels. The arithmetic crossover operator can keep the high-ranking individuals in parent generation and increase the low-ranking individuals in offspring population. Therefore, the quality of offspring population and the diversity of whole population shall be improved. For two given parent vectors $P_{1}=\left(v_{1}^{(1)}, v_{2}^{(1)}, \cdots, v_{m}^{(1)}\right)$ and $P_{2}=\left(v_{1}^{(2)}, v_{2}^{(2)}, \cdots, v_{m}^{(2)}\right)$, two offspring $C z=\left(z_{1}, z_{2}, \ldots, z_{m}\right)$ and $C_{w}=\left(w_{1}, w_{2}, \ldots, w_{m}\right)$ can be obtained by the designed arithmetic crossover operator:

$$
\left\{\begin{array}{l}
z_{i}=\alpha_{i} v_{i}^{(1)}+\left(1-\alpha_{i}\right) v_{i}^{(2)}, \\
w_{i}=\alpha_{i} v_{i}^{(2)}+\left(1-\alpha_{i}\right) v_{i}^{(1)},
\end{array}\right.
$$

where $v_{i}^{(1)}$ and $v_{i}^{(2)}$ indicate the $i$-th parent individual of the first and second generations; $z_{i}$ and $w_{i}$ are the child individual; $i=1,2, \ldots, m$; and $\alpha_{i}$ is the random number in $[0,1]$, which is expanded as

$$
\alpha_{i}=\left\{\begin{array}{l}
\frac{B_{r}}{A_{r}+B_{r}}, A_{r} \neq B_{r}, \\
\frac{A_{d}}{A_{d}+B_{d}}, A_{r}=B_{r},
\end{array}\right.
$$

where $A_{r}$ and $B_{r}$ indicate the nondominated sorting level of individual $A$ and individual $B$, respectively and $A_{d}$ and $B_{d}$ are the crowding distance of individual $A$ and individual $B$, respectively.

To change the disadvantage of low convergence speed caused by polynomial mutation parameters, a new Poisson distribution mutation operator is proposed, which can overstep the local optimum and thereby is conducive to search for global solutions. Assuming that the individual before mutation is expressed as $x=\left(x_{1}, x_{2}, \ldots, x_{\mathrm{n}}\right)$ and the individual after compilation is expressed as $x I=\left(x_{1}^{\prime}, x_{2}^{\prime}, \cdots, x_{n}^{\prime}\right)$, then the Poisson mutation operator can be designed as

$$
x_{i}^{\prime}=x_{i}+\left(x_{i}^{u}-x_{i}^{l}\right) \delta_{k},
$$

where $x_{i}^{\prime}$ means the $i$-th child individual after mutation; $x_{i}$ is the $i$-th parent individual; $x_{u}^{i}$ and $x_{l}^{i}$ are the upper and bottom boundaries of gene value at the mutation point $i$, respectively; $i=1,2, \ldots, m$; and $\delta_{k}$ is the Poisson random number, which is introduced as

$$
\delta_{k}=\frac{\lambda^{k} e^{-\lambda}}{k !},
$$

where $\lambda$ denotes the distribution parameter and $k$ is the variable value.

To obtain the Pareto optimal frontier and acquire nondominated solutions, the DMOGA algorithm is proposed by designing the arithmetic crossover operator and Poisson mutation operator. The essential process of DMOGA algorithm is summarized as follows:

(1) Generate initial $N$ individuals of population $P_{n}$, and set iteration times $n=0$

(2) Evaluate fitness values of all individuals, and rank them with Pareto dominance and crowding distances

(3) Select individuals from $P_{n}$ by the binary tournament method

(4) Obtain the child $C_{n}$ by the arithmetic crossover operator and Poisson mutation operator

(5) Generate population $Q_{n}$ by merging parent and child individuals, evaluate fitness value of all individuals, and perform fast nondominated sorting of $Q_{n}$

(6) Calculate crowding degree and crowding distance, and select individuals to form the new species group $P_{n+1}$

(7) Terminate the algorithm when the accuracy requirement is met; otherwise, the algorithm will back to $(2)$

(8) Acquire Pareto optimal surface and global optimal solution

By absorbing the global search capability of the arithmetic crossover operator and fast convergence ability of the Poisson mutation operator, the proposed DMOGA can accurately solve the multiobjective design optimization model and quickly obtain the Pareto solution set. To achieve the tradeoff effect between multiple objectives and optimal solution, a Utopia-Pareto directing adaptive (UPDA) search scheme is adopted by capturing plenty of characteristics and utilizing the ordering information of Pareto solutions $[35,44]$, which is conducive to find the best optimal solution for multiobjective design optimization of the multicomponent system.

2.3. Multiobjective Design Optimization Framework with $D C O K M$ and DMOGA. For the multicomponent system optimal design problem, we develop a multiobjective design optimization framework based on a surrogate model (i.e., DCOKM) to approximate multiple responses and an 


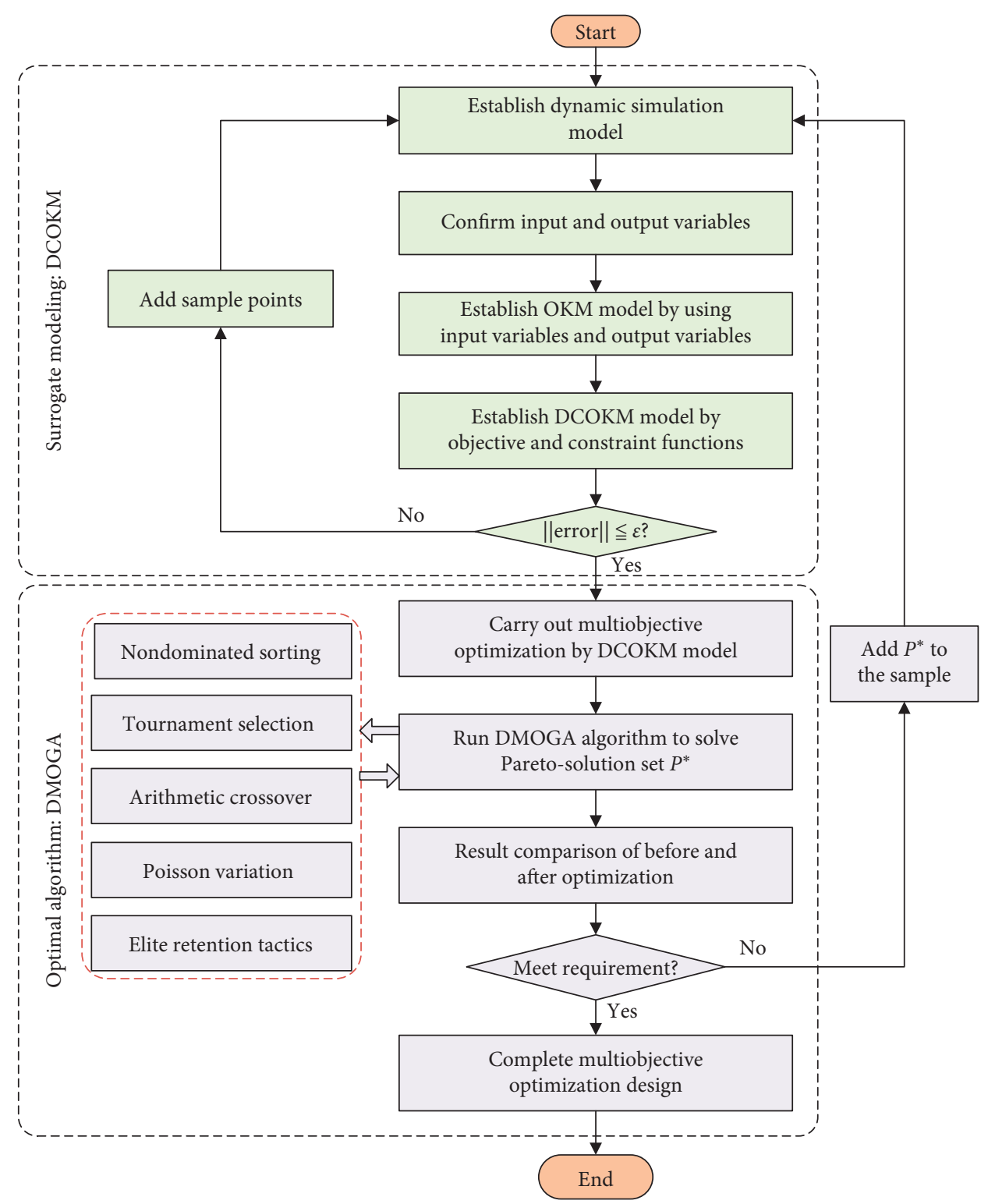

Figure 2: Multiobjective design optimization framework.

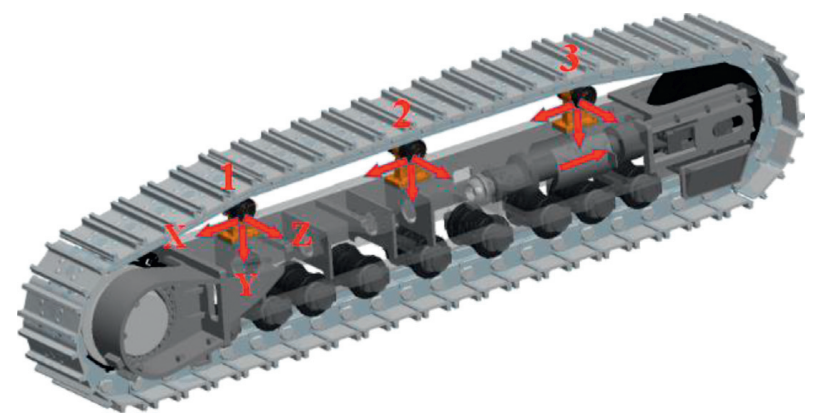

FIGURE 3: Load transfer path of the multicomponent system.

optimal algorithm (i.e., DMOGA) to acquire optimal solution set. Herein, the improved PSO algorithm and Kriging model are first absorbed into decomposed collaborative strategy, to enhance the approximation efficacy of the surrogate model; then, the arithmetic crossover operator and Poisson mutation operator are designed, to acquire the efficient and accurate convergence of the optimal algorithm. Therefore, by combining the DCOKM and DMOGA, the 


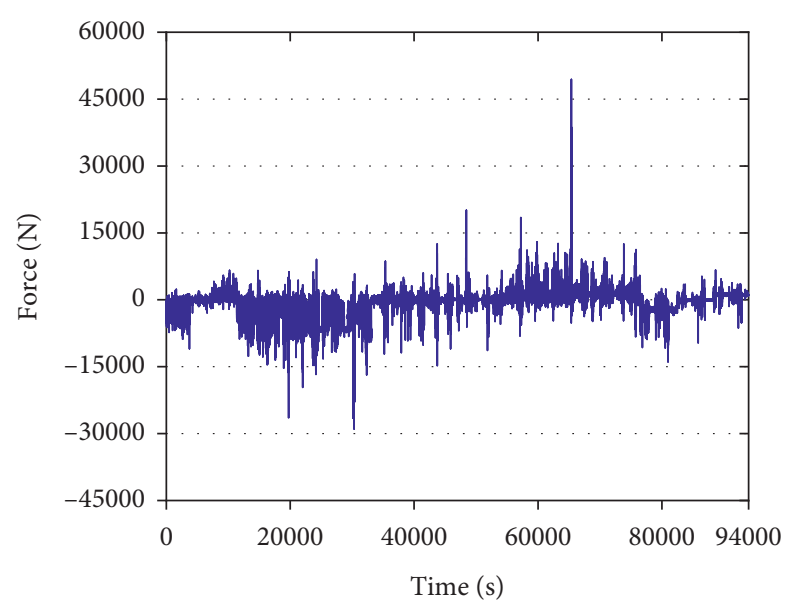

(a)

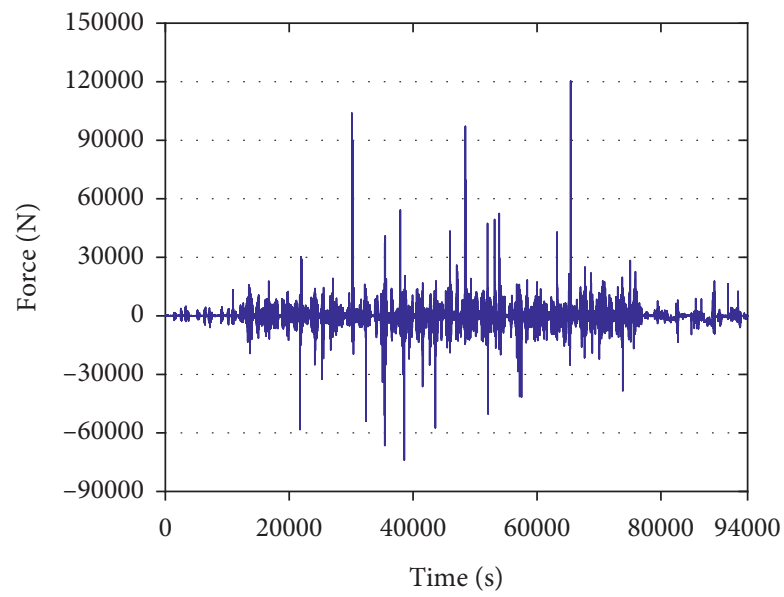

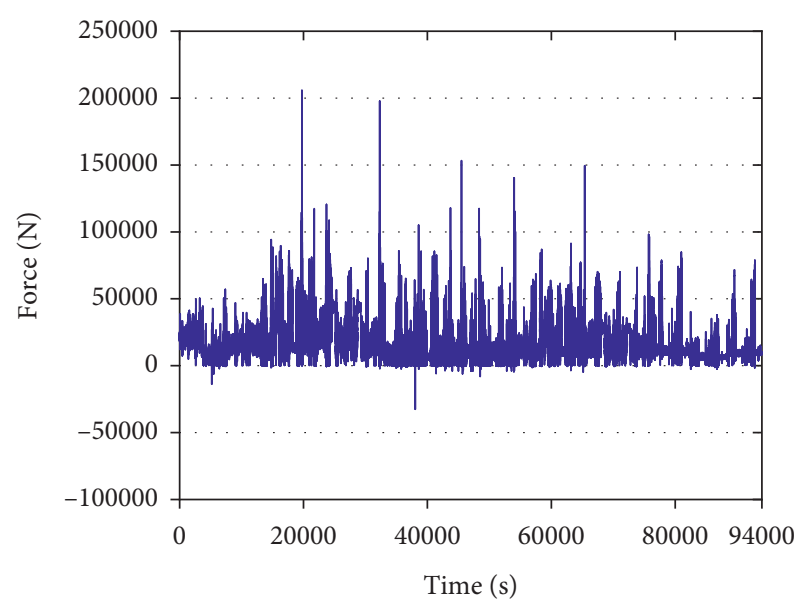

(b)

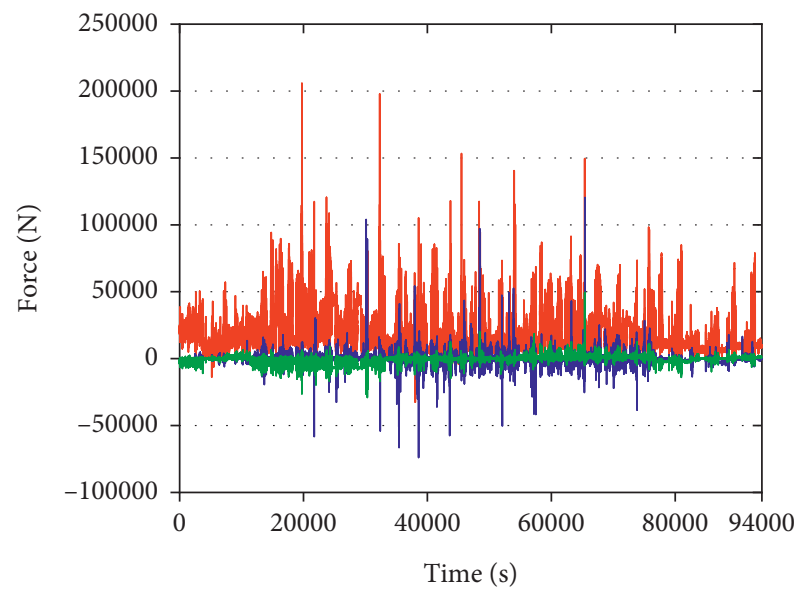

$X$
$-Y$
$-Z$

(c)

(d)

FIgURE 4: Load analysis of the first carrier roller: (a) forward force $X$, (b) radial force $Y$, (c) axial force $Z$, and (d) three directional forces.

calculation accuracy and efficiency of the multiobjective design optimization model is promising to be greatly improved. The multiobjective design optimization framework is shown in Figure 2.

\section{Case Study}

In this section, a carrier roller system from the track driving device of the excavator is selected as an engineering case to verify the effectiveness of the presented multiobjective design optimization framework. It should be noted that all computations are performed on an Inter(R) Core (TM) Desktop Computer (i7-9700K CPU $3.6 \mathrm{GHz}$ and $16 \mathrm{~GB}$ RAM).

3.1. Multiobjective Optimal Modeling. A typical carrier roller system mainly includes three components, and each component endures multidirectional nonuniform loading. The change of structural size for one component will cause the change of the contact force between the whole track and the roller, and then influence the load of other components, which will lead to the load change among the multiple component systems. The load transfer path of the carrier roller system is shown in Figure 3. During the working process, each carrier roller bears the load influence of three directions at the same time, and the load fluctuation in each direction is indicated in Figure 4. To confirm the important influencing factors of complex nonuniform loading, the load distribution of the carrier roller system is quantified in Figures 5-8. It reveals that the resultant force of three components shows V-shape distribution. Moreover, to further find the reasons of the great dispersion on resultant force, the load distribution of standard deviation in each direction is investigated and illustrated in Figures 9-11. Meanwhile, the stiffness analysis of the carrier roller system is performed and the results are depicted in Figure 12. We observe that with the increase of stiffness, the force standard 


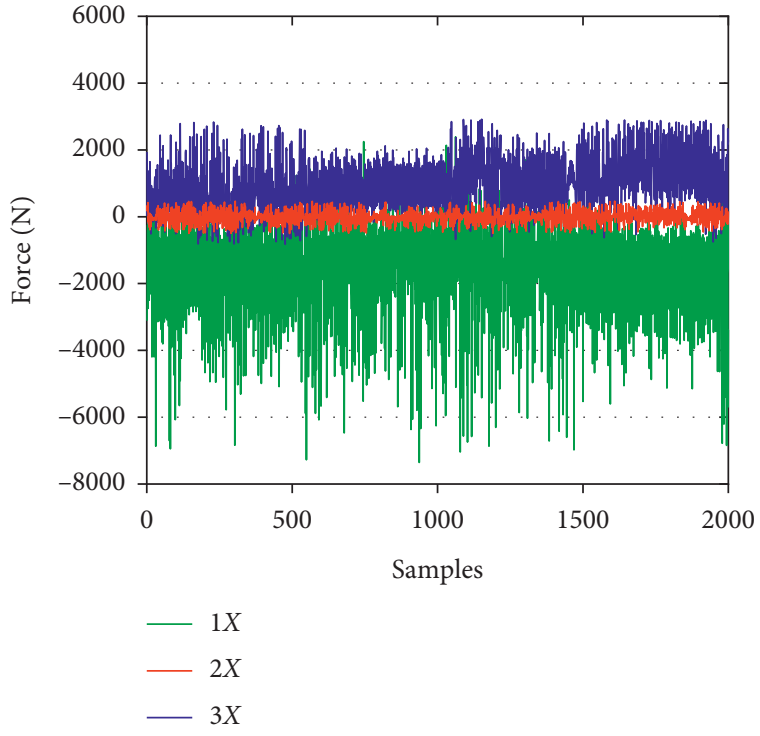

(a)

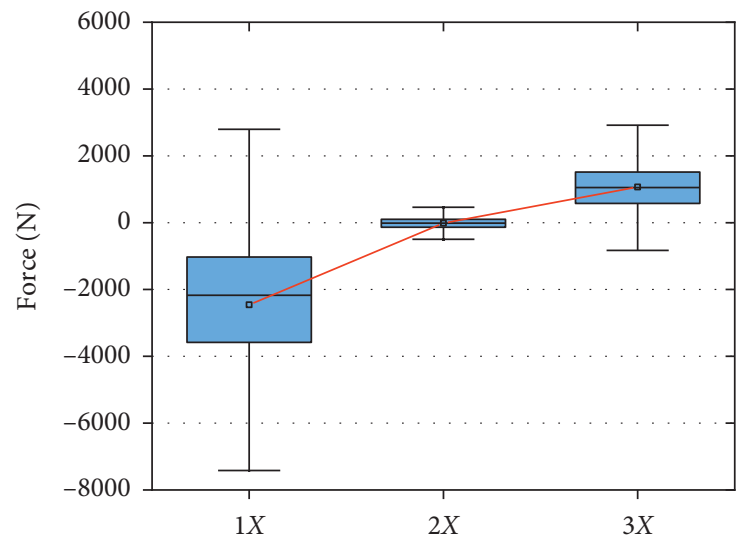

Figure 5: Load distribution of forward force $X$ of the carrier roller system: (a) simulation history and (b) boxplot curve.

TABLE 1: Extracted training samples.

\begin{tabular}{|c|c|c|c|c|c|c|c|c|}
\hline Sample & $x_{1}$ & $x_{2}$ & $x_{3}$ & $\sigma_{F 1}$ & $\sigma_{F 3}$ & $\sigma_{M F}$ & $S_{M F}$ & $S_{F \max }$ \\
\hline 1 & 390 & 39 & 446 & 5478.36 & 6088.866 & 2096.75 & 34708.48 & 70669.73 \\
\hline 2 & 433 & 106 & 462 & 6752.66 & 6670.62 & 4389.78 & 49454.32 & 93768.56 \\
\hline 3 & 409 & 48 & 406 & 4213.39 & 4146.539 & 4882.84 & 34017.66 & 63289.52 \\
\hline 4 & 418 & 82 & 453 & 5718.21 & 5777.563 & 1403.27 & 43320.44 & 85526.51 \\
\hline 5 & 393 & 86 & 459 & 6297.80 & 6242.555 & 2301.85 & 41561.77 & 89275.85 \\
\hline 6 & 449 & 91 & 448 & 5431.01 & 5322.861 & 5860.59 & 47852.8 & 89216.56 \\
\hline 7 & 424 & 140 & 451 & 8671.58 & 7605.778 & 2878.48 & 51373.97 & 113861.80 \\
\hline 8 & 473 & 108 & 488 & 7110.48 & 6871.813 & 12331.36 & 57903.74 & 112841.70 \\
\hline 9 & 485 & 76 & 470 & 6382.03 & 6362.146 & 11143.59 & 52054.45 & 93507.65 \\
\hline 10 & 457 & 128 & 409 & 4517.62 & 5344.153 & 10078.74 & 49212.41 & 112440.51 \\
\hline 11 & 451 & 23 & 432 & 4282.40 & 5153.78 & 4734.30 & 37107.03 & 76721.86 \\
\hline 12 & 423 & 55 & 443 & 6500.60 & 5537.399 & 1825.19 & 39707.02 & 95494.29 \\
\hline 13 & 481 & 123 & 400 & 3658.48 & 4148.80 & 13708.95 & 51039.61 & 99463.75 \\
\hline 14 & 458 & 98 & 403 & 3675.75 & 3830.37 & 9571.45 & 44760.41 & 85159.23 \\
\hline 15 & 441 & 60 & 485 & 6235.71 & 5748.82 & 6150.34 & 46056.87 & 111631 \\
\hline 16 & 479 & 136 & 450 & 8958.53 & 8488.76 & 12654.48 & 58537.90 & 119643 \\
\hline 17 & 472 & 31 & 395 & 2550.09 & 3079.38 & 8237.67 & 36571.72 & 65595.75 \\
\hline 18 & 462 & 69 & 464 & 6185.57 & 6892.61 & 7400.77 & 47623.44 & 105196.80 \\
\hline 19 & 429 & 90 & 434 & 5715.60 & 6410.48 & 3553.80 & 43720.39 & 85185.63 \\
\hline 20 & 426 & 47 & 468 & 5295.95 & 5673.30 & 2924.82 & 40827.15 & 77965.02 \\
\hline 21 & 414 & 107 & 392 & 2459.88 & 3156.21 & 8467.26 & 39682.11 & 78960.68 \\
\hline 22 & 413 & 30 & 452 & 4183.20 & 4876.16 & 520.430 & 36114.36 & 63734.86 \\
\hline 23 & 456 & 56 & 397 & 4436.30 & 4444.57 & 7942.65 & 38563.35 & 83004.92 \\
\hline 24 & 428 & 103 & 487 & 6741.81 & 7158.78 & 6310.21 & 51060.07 & 95940.30 \\
\hline 25 & 477 & 24 & 465 & 4936.89 & 6018.77 & 7260.10 & 41893.87 & 72833.50 \\
\hline 26 & 399 & 129 & 472 & 9084.01 & 6987.26 & 3386.82 & 49349.60 & 117109.30 \\
\hline 27 & 475 & 26 & 440 & 4903.20 & 6094.30 & 6697.05 & 39920.45 & 67536.79 \\
\hline 28 & 430 & 97 & 410 & 4272.04 & 5065.08 & 6294.49 & 42083.32 & 87140.54 \\
\hline 29 & 431 & 57 & 398 & 3964.19 & 4139.24 & 6453.212 & 36342.50 & 70549.19 \\
\hline 30 & 480 & 63 & 404 & 5216.05 & 6164.22 & 10156.87 & 42814.12 & 120803.00 \\
\hline
\end{tabular}




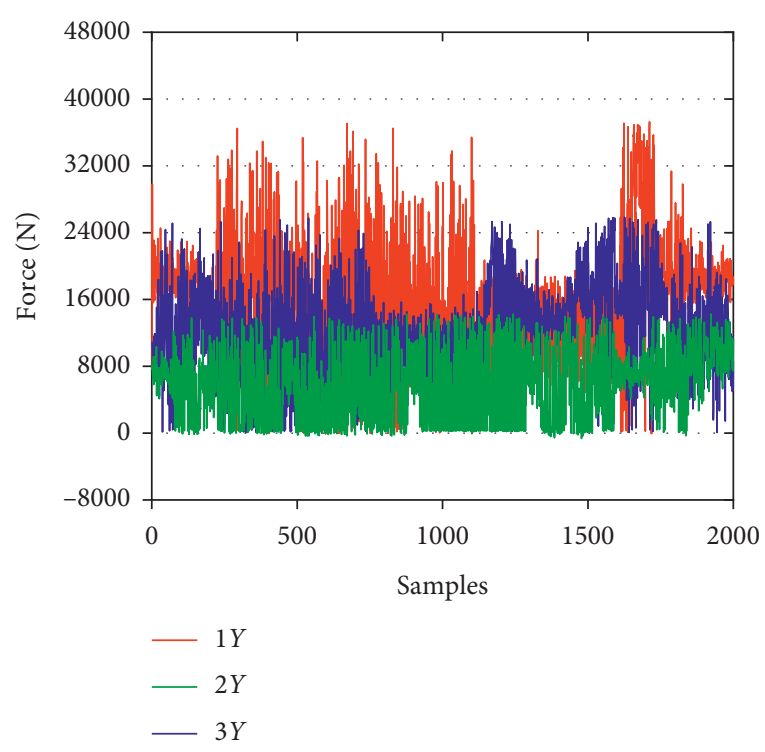

(a)

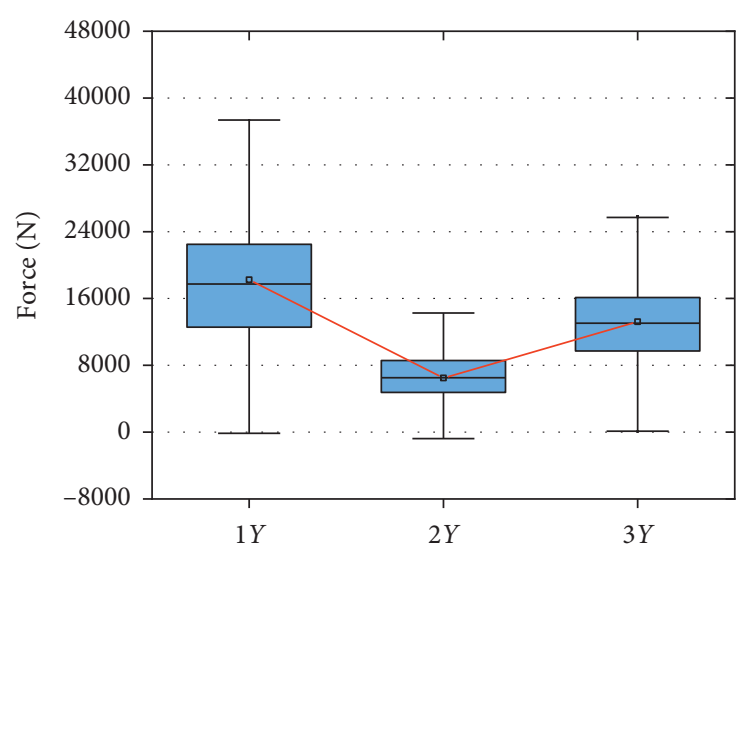

(b)

Figure 6: Load distribution of radial force $Y$ of the carrier roller system: (a) simulation history and (b) boxplot curve.

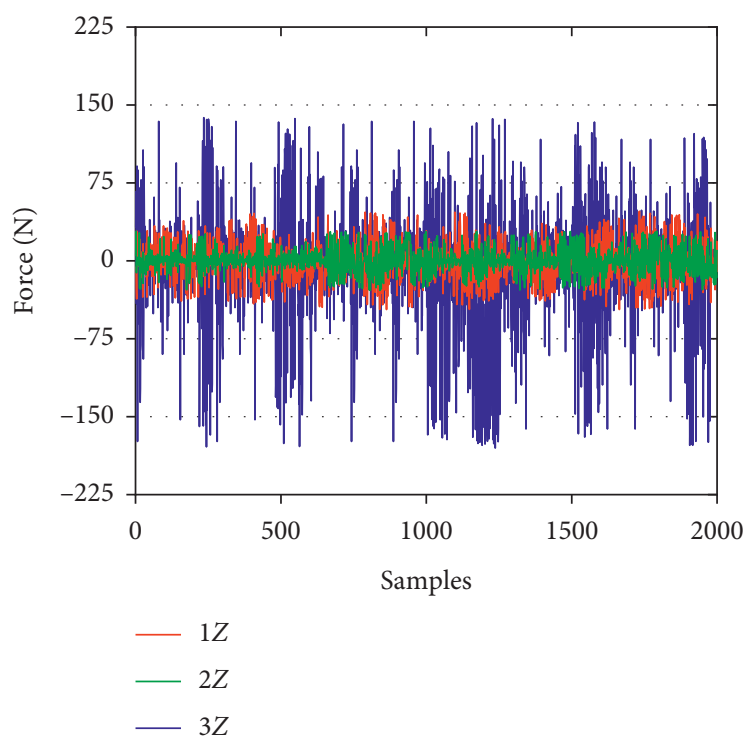

(a)

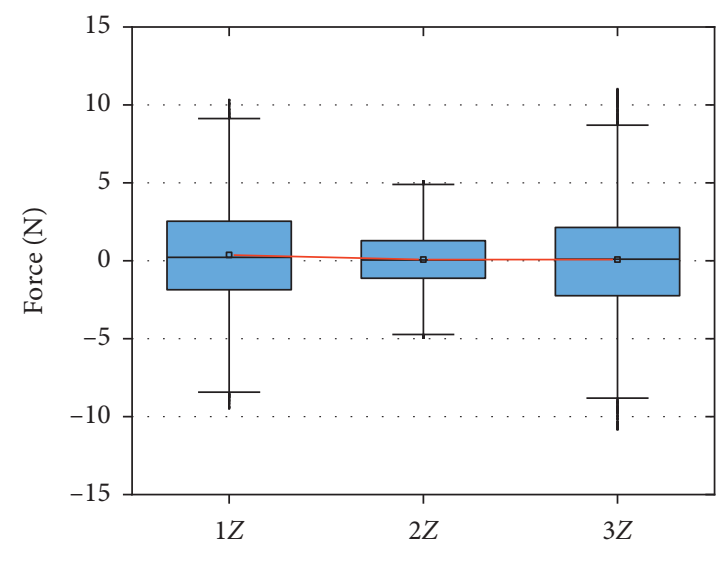

(b)

FIgURE 7: Load distribution of axial force $Z$ of the carrier roller system: (a) simulation history and (b) boxplot curve.

deviation in the first component $\sigma_{F 1}$, in the third component $\sigma_{F 3}$, and in all rollers $\sigma_{M F}$ (i.e., the sum of maximum force) appears significant fluctuations, while the sum of mean force in all rollers $S_{M F}$ and the sum of maximum force in all rollers $S_{F \max }$ show little variations.

Therefore, by minimizing the responses of $\sigma_{F 1}, \sigma_{F 3}$, and $\sigma_{M F}$, the nonuniform appearance would be reduced and the load distribution will become more uniform. Therefore, to minimize the nonuniform loading of the multicomponent system, by regarding the force standard deviations (i.e., $\sigma_{F 1}$, $\sigma_{F 3}$, and $\sigma_{M F}$ ) as optimal objectives, force mean values (i.e., $S_{M F}$ and $S_{F \max }$ ) and other constraints as constraint functions, and the structural sizes (i.e., height of first component $x_{1}$, height of third component $x_{3}$, and displacement condition $x_{2}$ ) as design variables, the multiobjective design optimization model is established as illustrated in the following equation: 


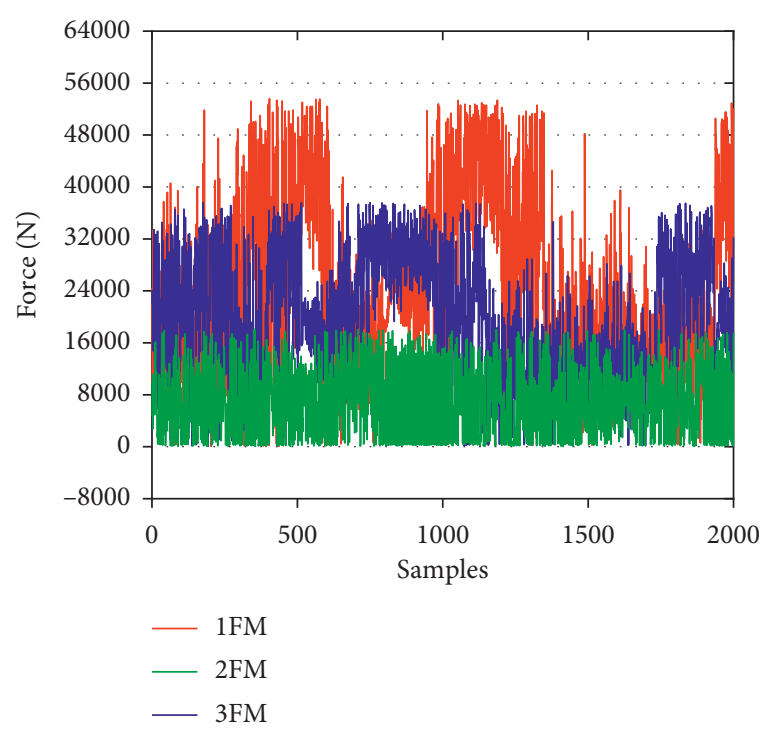

(a)

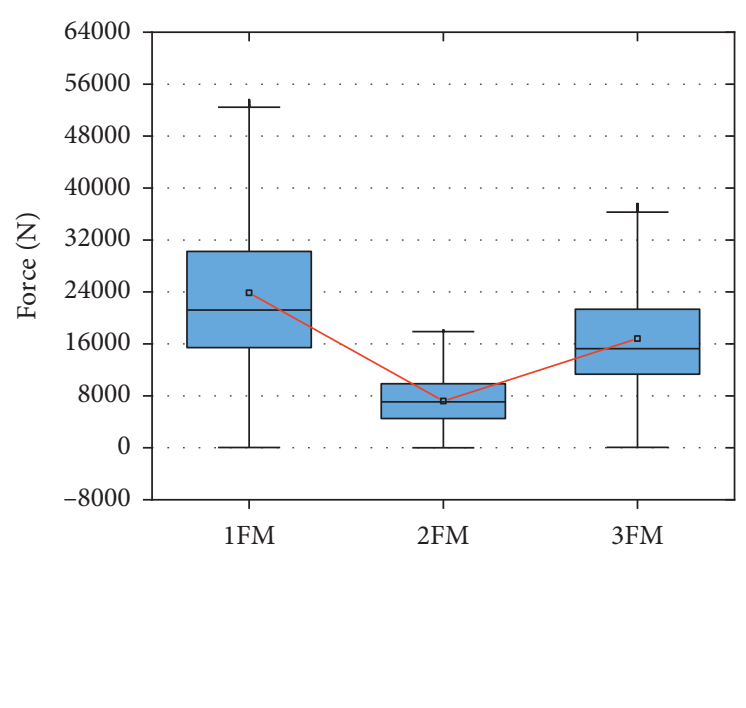

(b)

FIGURE 8: Load distribution of resultant force FM of the carrier roller system: (a) simulation history and (b) boxplot curve.

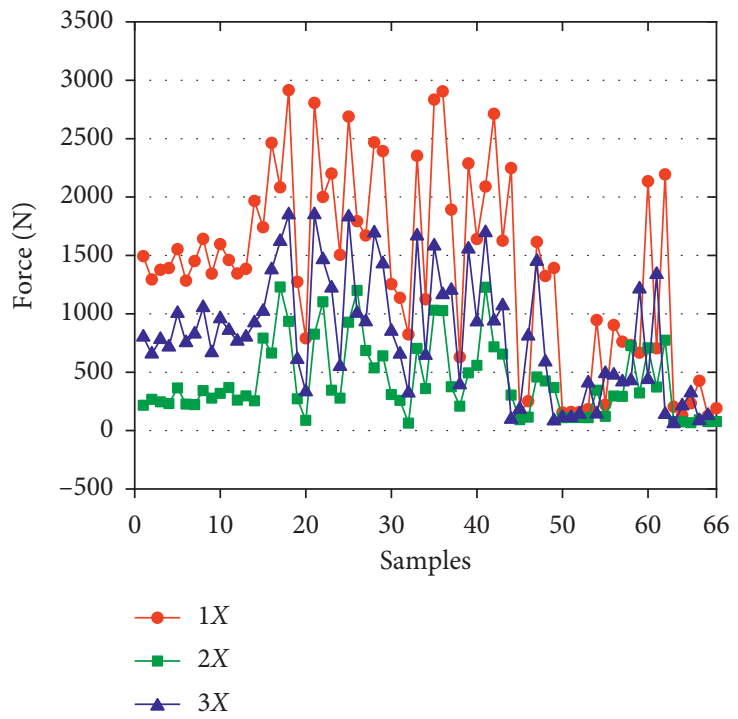

(a)

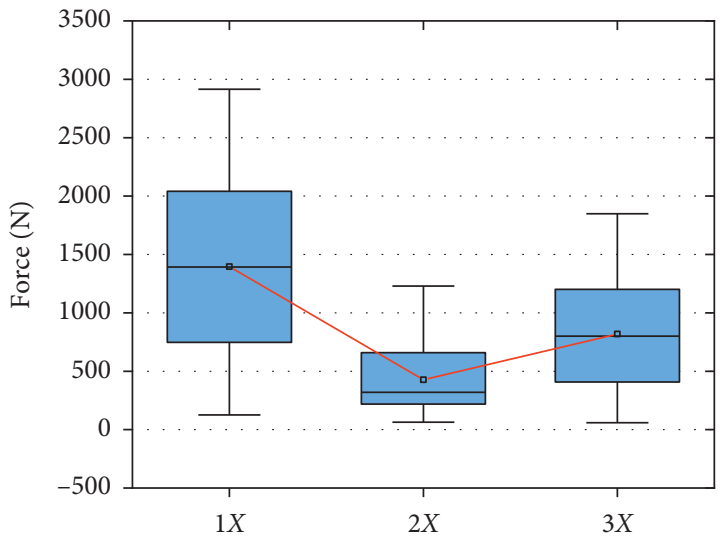

(b)

FIgURE 9: Load distribution of standard deviation $X$ of the carrier roller system: (a) simulation history. and (b) boxplot curve.

$$
\begin{aligned}
& \text { Find } x_{1}, x_{2}, x_{3} \text {, } \\
& \min \sigma_{F 1}(x)=\sigma_{F 1}\left(x_{1}, x_{2}, x_{3}\right), \\
& \min \sigma_{F 3}(x)=\sigma_{F 3}\left(x_{1}, x_{2}, x_{3}\right) \text {, } \\
& \min \sigma_{M F}(x)=\sigma_{M F}\left(x_{1}, x_{2}, x_{3}\right) \text {, } \\
& \text { S.t. }\left\{\begin{array}{l}
S_{M F}<\left[S_{M F}\right], \\
S_{F \max }<\left[S_{F \max }\right], \\
407.5 \leq x_{1} \leq 527.5, \\
10 \leq x_{2} \leq 70, \\
407.5 \leq x_{3} \leq 527.5,
\end{array}\right.
\end{aligned}
$$

\subsection{Surrogate Modeling by DCOKM}

3.2.1. Sample Generations. To obtain the objective responses and constraint function values of the multiobjective design optimization model, the kinematic and dynamic equations are solved as follows.

Based on Lagrange multiplier modeling technique $[45,46]$, the dynamic equation of the carrier roller system in the generalized coordinate system is established as 


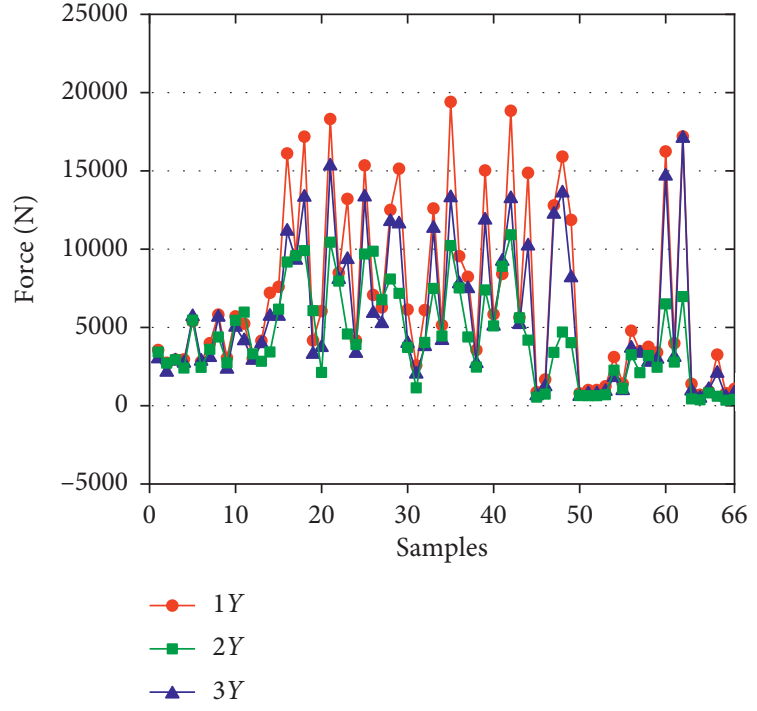

(a)

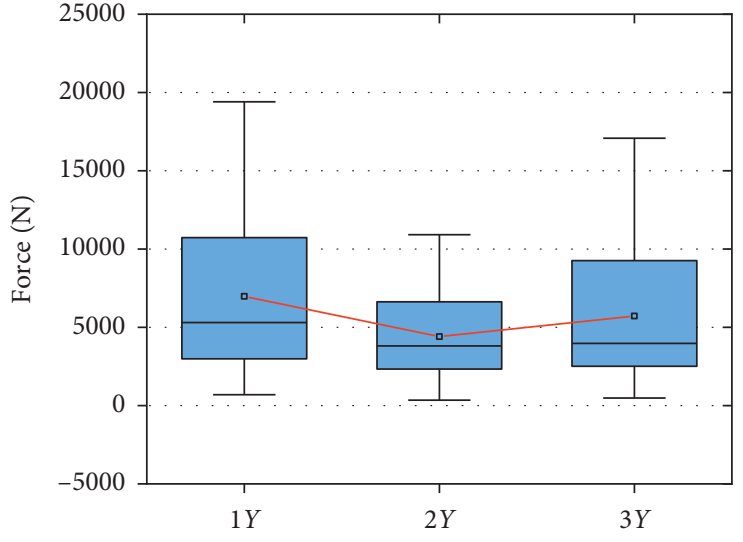

(b)

Figure 10: Load distribution of standard deviation $Y$ of the carrier roller system: (a) simulation history and (b) boxplot curve.

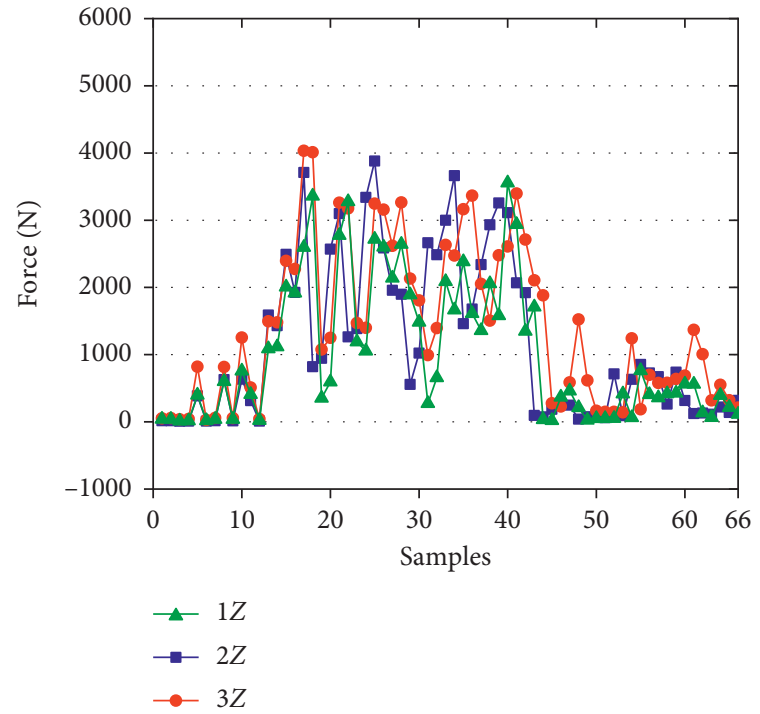

(a)

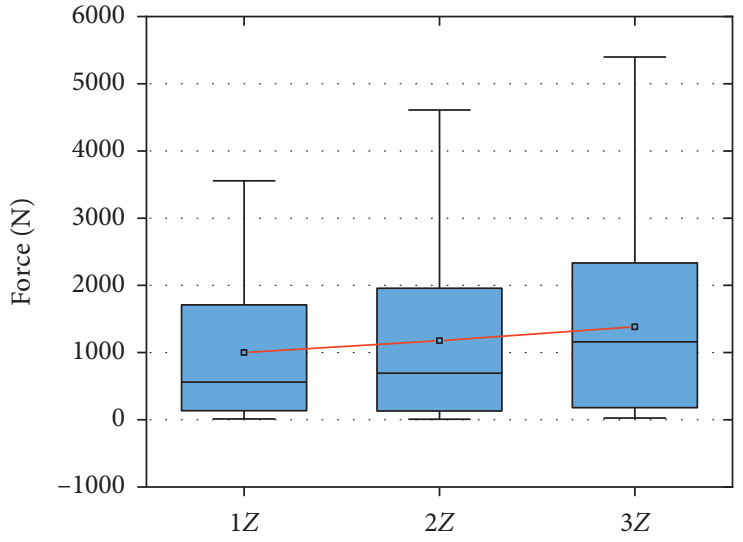

(b)

FIGURE 11: Load distribution of standard deviation $Z$ of the carrier roller system: (a) simulation history and (b) boxplot curve.

$$
\frac{d}{d t}\left(\frac{\partial T}{\partial \dot{q}}\right)-\left(\frac{\partial T}{\partial q}\right)^{T}+f_{q}^{T} \rho+g_{\dot{q}}^{T} \mu-Q=0,
$$

where $T$ denotes the system kinetic energy; $q$ is the generalized coordinates, which is related to the variable $x_{1}, x_{2}$, and $x_{3} ; \dot{q}$ is the generalized velocities, which is related to the variable $x_{1}, x_{2}$, and $x_{3} ; Q$ is the generalized forces; $\rho$ is the Lagrange multiplier array under holonomic constraints; and $\mu$ is the Lagrange multiplier array under nonholonomic constraints. Herein, the holonomic and nonholonomic constraints in (18) are expressed as

$$
\left\{\begin{array}{l}
f(q, t)=0 \\
g(q, \dot{q}, t)=0
\end{array}\right.
$$

Subsequently, by solving the constraint equation with the gear prediction-correction algorithm, the velocity and acceleration at time $t_{n}$ can be obtained as 


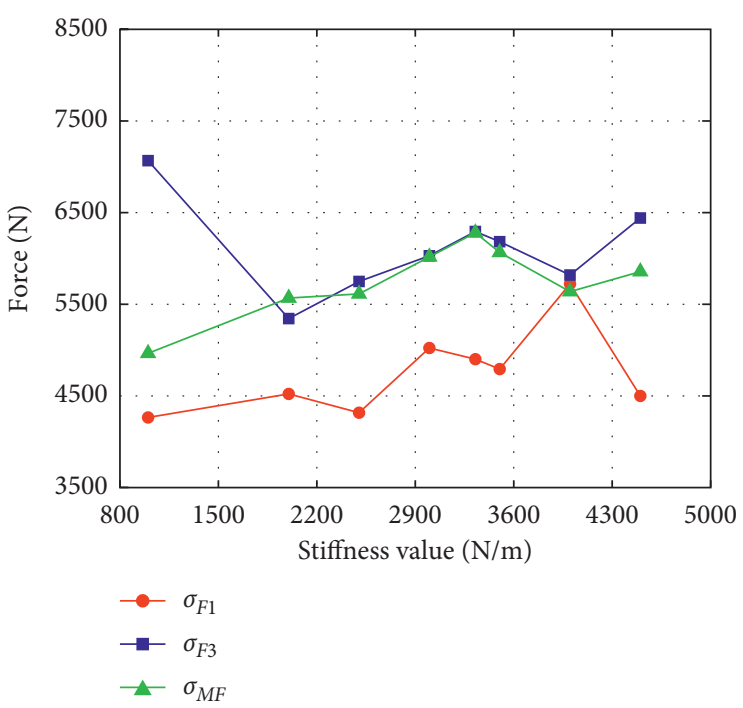

(a)

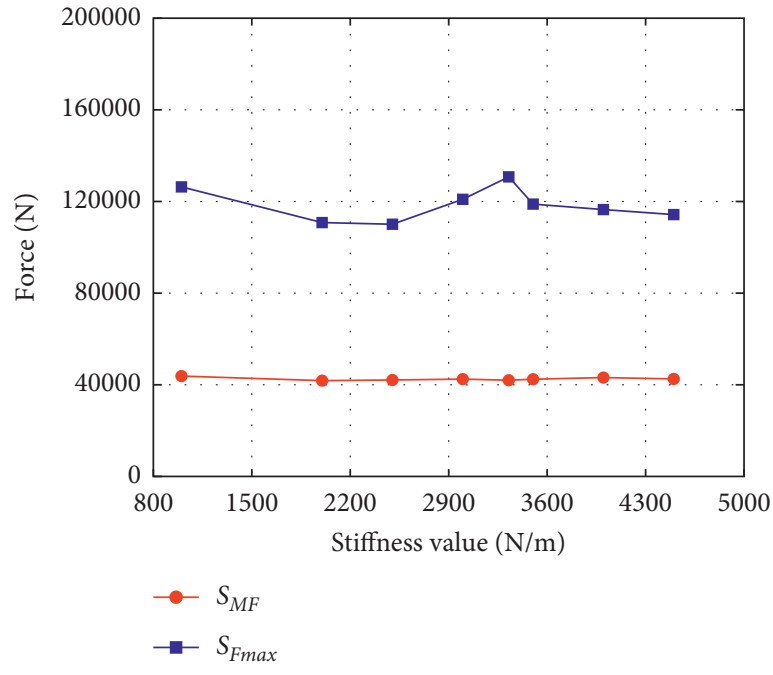

(b)

FIGURE 12: Load fluctuation with different stiffness value. (a) Force standard deviations. (b) Sum of mean force and maximum force.

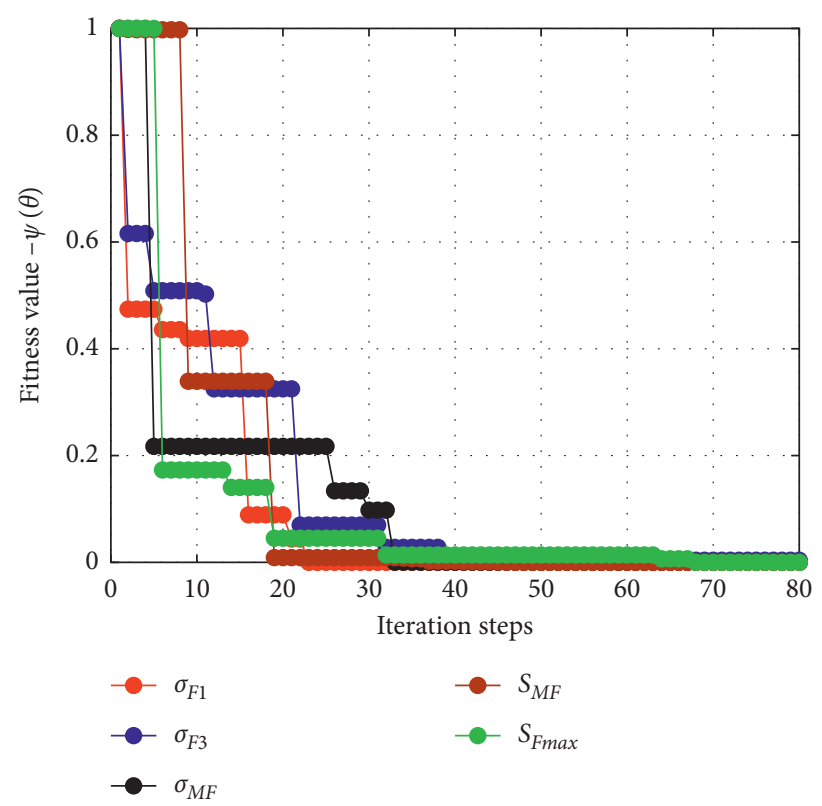

Figure 13: Training process of DCOKM.

TABLE 2: Optimal correlation parameter of DCOKM.

\begin{tabular}{lccr}
\hline Response & \multicolumn{2}{c}{ Related parameters $\theta^{*}$} \\
& $\theta_{1}$ & $\theta_{2}$ & $\theta_{3}$ \\
\hline$\sigma_{F 1}$ & 38.83488 & 40.38819 & 42.71815 \\
$\sigma_{F 3}$ & 3.086433 & 3.201069 & 3.394138 \\
$\sigma_{M F}$ & 48.52073 & 50.46128 & 53.37277 \\
$S_{M F}$ & 49.97663 & 51.97569 & 54.97429 \\
$S_{F \max }$ & 49.98934 & 51.98891 & 54.98827 \\
\hline
\end{tabular}




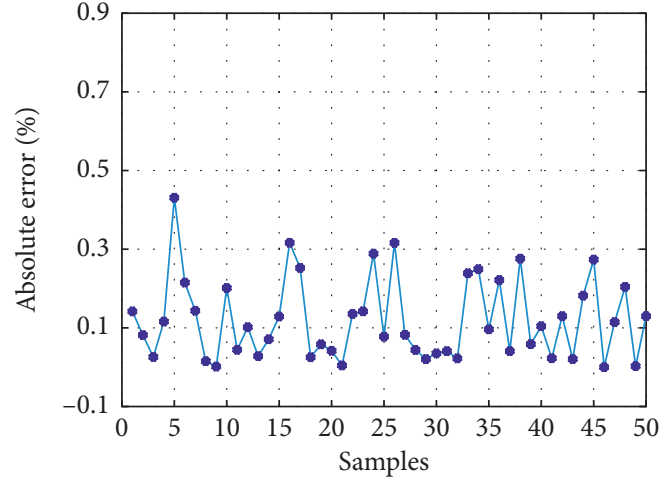

(a)

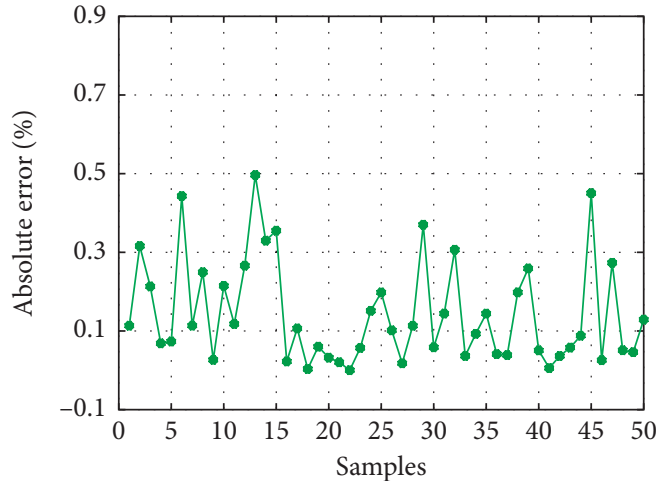

(b)

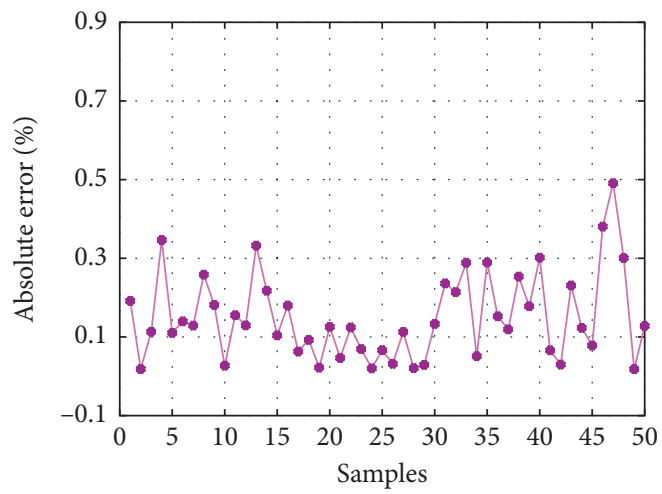

(c)

Figure 14: Absolute errors of three objectives: (a) $\sigma_{F 1}$, (b) $\sigma_{F 3}$, and (c) $\sigma_{M} F^{\prime}$.

TABle 3: Comparison of results before and after optimization.

\begin{tabular}{lcccc}
\hline Optimization index & Before optimization & After optimization & Reduce the proportion (\%) & Remark \\
\hline$x_{1}$ & 467 & 412 & - & - \\
$x_{2}$ & 40 & 10 & - & - \\
$x_{3}$ & 467 & 459 & - & - \\
$\sigma_{F 1}$ & 8735 & 4382 & 67 & Reduce track run out and impact load \\
$\sigma_{F 3}$ & 8156 & 924 & 46 & Reduce track run out and impact load \\
$\sigma_{M F}$ & 10987 & 33924 & 92 & Better load uniformity \\
$S_{M F}$ & 59013 & 57477 & 55 & Reduce wear failure rate \\
$S_{F \max }$ & 131842 & 43 & Reduce fracture failure rate \\
\hline
\end{tabular}

TABle 4: Approximation efficacy of different models.

\begin{tabular}{lcccc}
\hline \multirow{2}{*}{ Methods } & \multicolumn{2}{c}{ Fitting surrogate models } & \multicolumn{2}{c}{ Testing surrogate models } \\
& Fitting number & Fitting time (s) & Testing number & Testing time (s) \\
\hline MCS & - & - & - & - \\
RS & 245 & $2.16 \times 10^{7}$ & 200 & 15.623 \\
KM & 180 & $1.55 \times 10^{7}$ & 200 & 13.532 \\
OKM & 130 & $1.12 \times 10^{7}$ & 200 & 11.367 \\
DCOKM & 90 & $7.78 \times 10^{6}$ & 200 & 9.9451 \\
\end{tabular}


TABLE 5: Computing efficiency and accuracy of different algorithms.

\begin{tabular}{lcccccccccc}
\hline \multirow{2}{*}{ Methods } & \multicolumn{3}{c}{ Before optimization $\left(\times 10^{4} \mathrm{~N}\right)$} & \multicolumn{3}{c}{ After optimization $\left(\times 10^{4} \mathrm{~N}\right)$} & \multicolumn{3}{c}{ Reduction } & \multicolumn{3}{c}{ Time $(\mathrm{s})$} \\
& $Y_{1}$ & $Y_{2}$ & $Y_{3}$ & $Y_{1}$ & $Y_{2}$ & $Y_{3}$ & $Y_{1}$ & $Y_{2}$ & $Y_{3}$ \\
& 0.8735 & 0.8156 & 1.0987 & 0.3332 & 0.4620 & 0.1023 & 0.61 & 0.43 & 0.90 & 18.23 \\
NCGA & 0.8735 & 0.8156 & 1.0987 & 0.3146 & 0.4657 & 0.1275 & 0.64 & 0.43 & 0.88 \\
NGGA & 0.8735 & 0.8156 & 1.0987 & 0.3065 & 0.4640 & 0.1112 & 0.65 & 0.43 & 0.89 & 16.52 \\
DMOGA & 0.8735 & 0.8156 & 1.0987 & 0.2904 & 0.4382 & 0.0924 & 0.67 & 0.46 & 0.92 & 13.31 \\
\hline
\end{tabular}

Note. $Y_{1}$ indicates the $\sigma_{F 1}, Y_{2}$ indicates the $\sigma_{F 3}$, and $Y_{3}$ indicates the $\sigma_{M F}$.

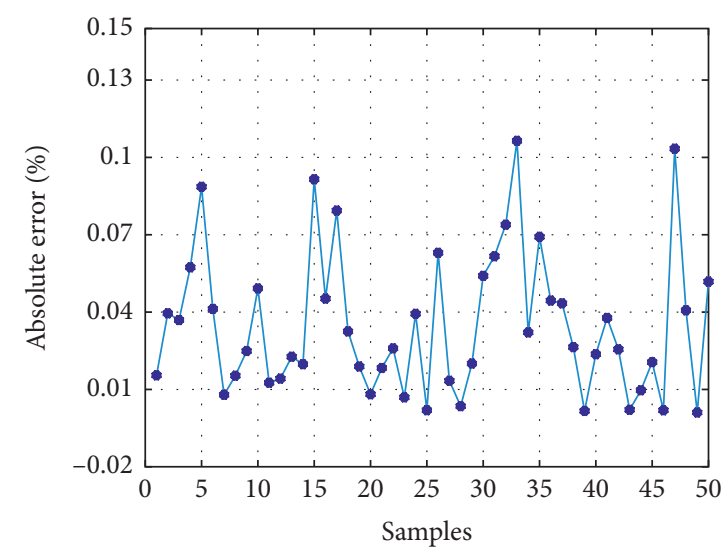

(a)

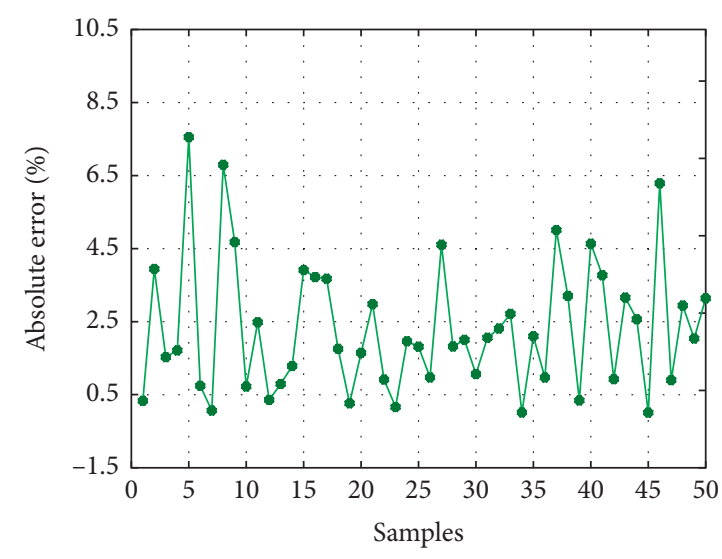

(b)

Figure 15: Absolute errors of two constraints: (a) $S_{M F}$ and (b) $S_{F m a x}$.

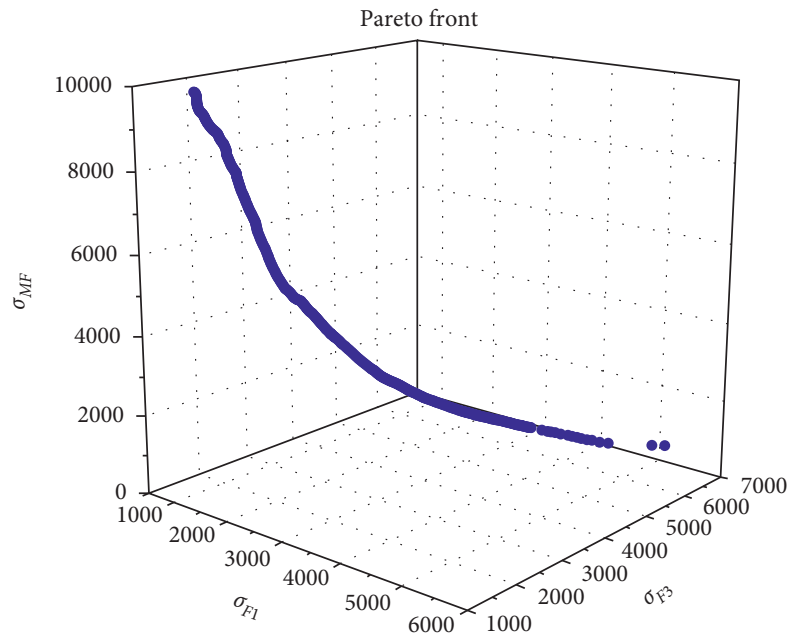

FIgURE 16: Pareto optimal frontier of MODO.

$$
\begin{aligned}
& \left(\frac{\partial \phi}{\partial q}\right) \dot{q}=-\frac{\partial \phi}{\partial t} \\
& \left(\frac{\partial \phi}{\partial q}\right) \ddot{q}=-\left\{\frac{\partial^{2} \phi}{\partial t^{2}}+\sum_{k=1}^{n} \sum_{l=1}^{n} \frac{\partial^{2} \phi}{\partial q_{k} \partial q_{l}} \dot{q}_{k} \dot{q}_{l}+\frac{\partial}{\partial t}\left(\frac{\partial \phi}{\partial q}\right) \dot{q}+\frac{\partial}{\partial q}\left(\frac{\partial \phi}{\partial t}\right) \dot{q}\right\}
\end{aligned}
$$

where $q_{k}$ and $q_{l}$ are the generalized coordinates of the $k$-th and $l$-th iterations, respectively, and $\dot{q}_{k}$ and $\dot{q}_{l}$ are the generalized velocities of the $k$-th and $l$-th iterations, respectively.
Consequently, by solving the Lagrange multiplier equation, the constraint and response reaction force in (18) are acquired:

$$
\left(\frac{\partial \phi}{\partial q}\right)^{T} \lambda=\left\{-\frac{d}{d t}\left(\frac{\partial T}{\partial \dot{q}}\right)^{T}+\left(\frac{\partial T}{\partial q}\right)^{T}+Q\right\} .
$$

Obviously, it is time-consuming to solving the nonlinear dynamic equations for thousands of times. Therefore, to build the surrogate model of the above dynamic equations, a 


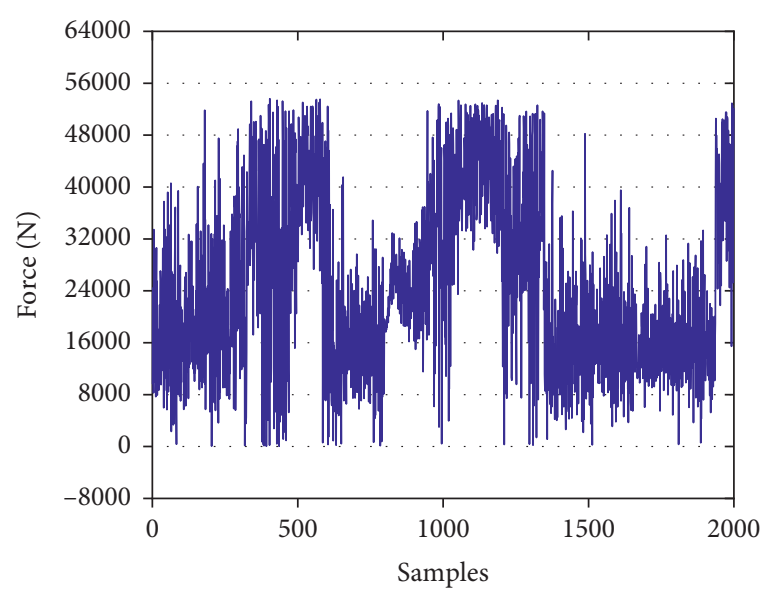

(1) $1 \mathrm{FM}$

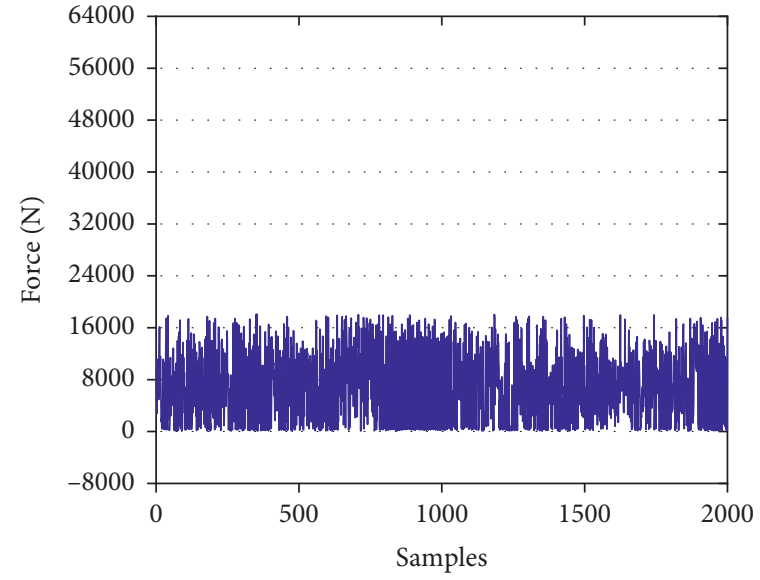

(2) $2 \mathrm{FM}$

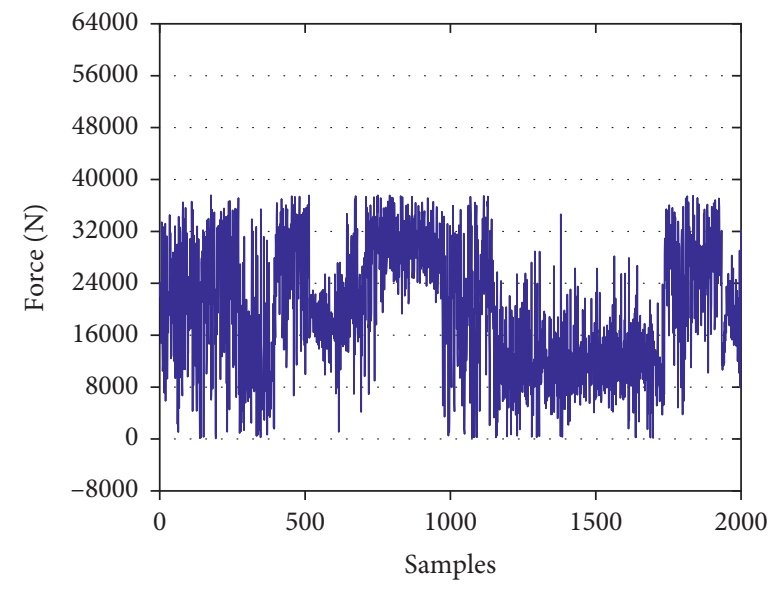

(3) $3 \mathrm{FM}$

(a)

Figure 17: Continued. 


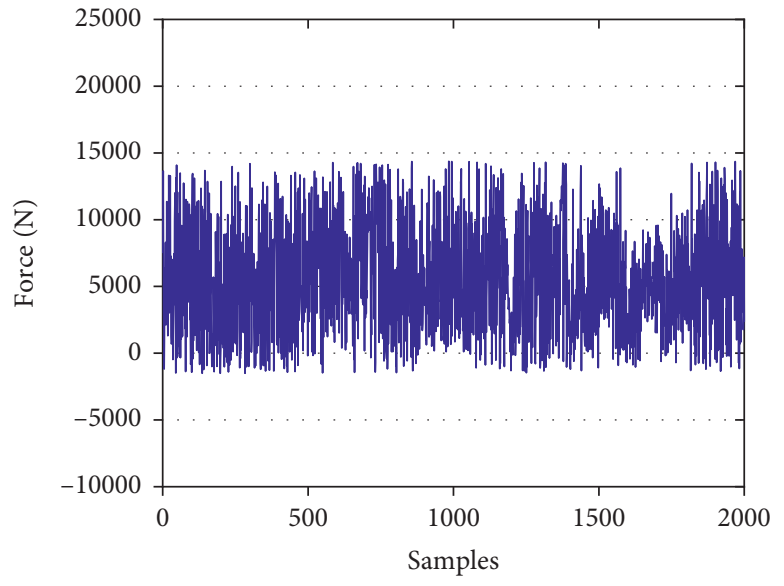

(1) $1 \mathrm{FM}$

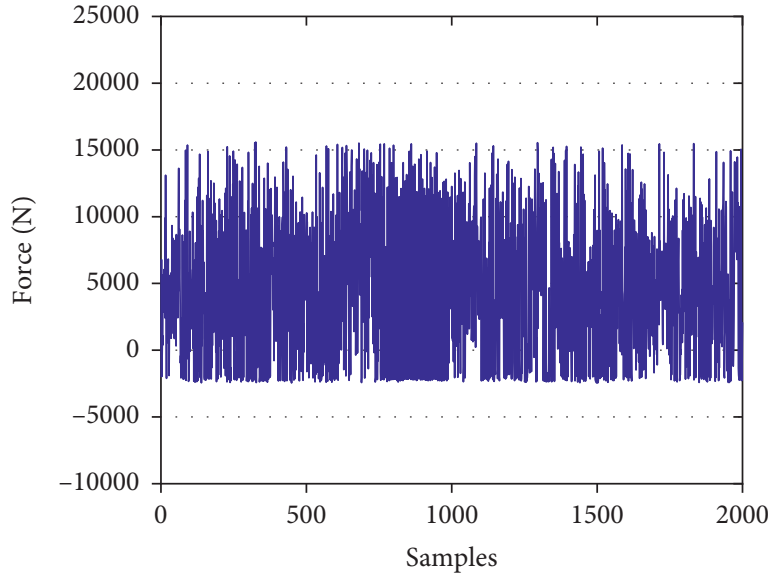

(2) $2 \mathrm{FM}$

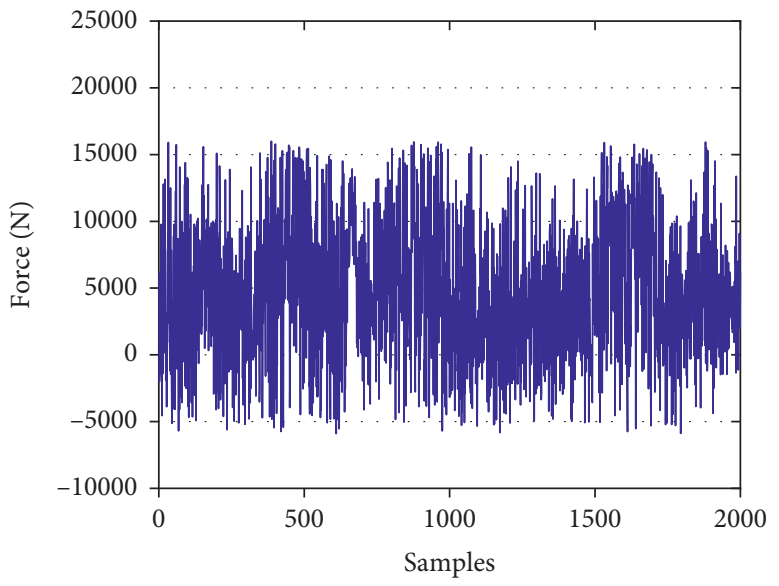

(3) $3 \mathrm{FM}$

(b)

FIGURE 17: Simulation history of three carrier rollers before and after optimization: (a) before optimization and (b) after optimization.

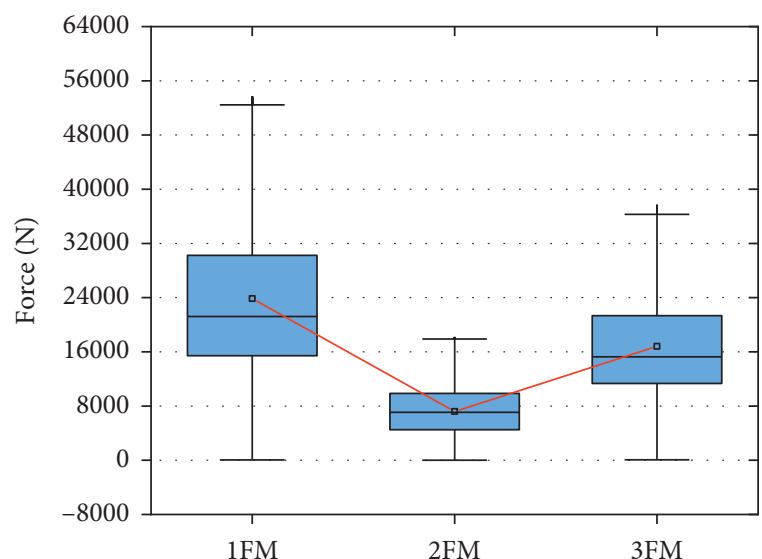

(a)

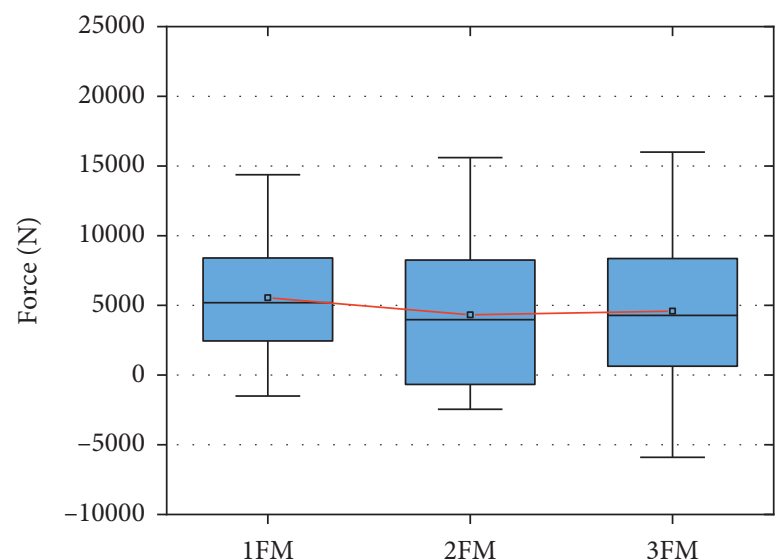

(b)

FIgURE 18: Resultant force of three carrier rollers before and after optimization: (a) before optimization and (b) after optimization.

handful of the input variables $\left(x_{1}, x_{2}\right.$, and $\left.x_{3}\right)$ are extracted by Latin hypercube sampling, and the corresponding output responses $\left(\sigma_{F 1}, \sigma_{F 3}, \sigma_{M F}, S_{M F}\right.$, and $\left.S_{F \max }\right)$ are obtained by running the above dynamic equations. The extracted samples would be considered as training samples for surrogate modeling, which are shown in Table 1. 
3.2.2. Fitting DCOKM. By importing the generated samples into the presented DCOKM method, we perform the surrogate modeling for multiobjective and multiconstraint. Considering the improved PSO algorithm, the optimal correlation parameter $\boldsymbol{\theta}^{*}$ is searched and substituted into OKM, and the DCOKM can be established. The optimal correlation parameter $\boldsymbol{\theta}^{*}$ of DCOKM is shown in Table 2. When the population size $N=100$, number of iteration $K=80$, maximum inertia weight $\omega_{\max }=0.9$, minimum inertia weight $\omega_{\min }=0.4$, initial individual learning factor $c_{1 s}=2.5$, individual learning factor in the largest iterations $c_{1 n}=0.5$, initial population learning factor $c_{2 s}=0.5$, and population learning factor in the largest iterations $c_{2 n}=2.5$, the training process of DCOKM is shown in Figure 13, which shows that the DCOKM modeling holds fast convergence speed and accuracy. Moreover, to verify the approximation accuracy of the established surrogate model, the absolute errors between the predicted objective/constraint and the actual objective/constraint are calculated as shown in Figures 14 and 15. It reveals that the absolute errors of the built DCOKM are nearly equal to zero, which is sufficient to meet the accuracy requirements of surrogate modeling.

3.3. Optimal Solution by DMOGA. By employing the built DCOKM to approximate the multiobjective and multiconstraint, the DMOGA is executed to solve the established MODO model shown in (18). Herein, the parent population size, crossover probability, mutation probability, and iteration number of DMOGA algorithm are set as 100, 0.9, 0.1, and 1000, respectively. After 1000 iterative times, the Pareto optimal frontier is obtained as shown in Figure 16. According to the Pareto curve in Figure 16, the optimization objectives $\sigma_{M F}$ and $\sigma_{F 1}$, and $\sigma_{M F}$ and $\sigma_{F 3}$ both show negative correlation and $\sigma_{F 1}$ and $\sigma_{F 3}$ show positive correlation. To achieve the reasonable balance between three objectives, we sort $\sigma_{F 1}$ and $\sigma_{F 3}$ as ascending order and $\sigma_{M F}$ as descending order. Then, the middle point in the Pareto front curve is selected as the optimal solution of multiobjective optimization $[35,44]$. In this case, the design variables $x_{1}, x_{2}$, and $x_{3}$ corresponding to the optimal solution are chosen as 412,10 , and 459 , respectively.

To validate the optimal results, we compare the optimal parameters before and after optimization in Table 3 , which shows that all objective responses are reduced after optimization, particularly the $\sigma_{M F}$ decreased by $92 \%$. To check the loading uniformity after optimization, we further compare the loading distribution before and after optimization in Figures 17 and 18. The loading distribution results indicate that the system loads are significantly reduced and the loading after optimization becomes more uniform than that of before optimization. Moreover, through the multiobjective design optimization of the carrier roller system, the average failure ratio of track driving device is reduced from $31 \%$ to $8 \%$.

3.4. Validations of Methods. Because of the particularity of the study case (practical engineering problem), there is no standard solution for reference. To compare the effectiveness of the proposed method, some advanced surrogate models and optimization algorithms are selected to perform the multiobjective design of the carrier roller system and then compared with the presented model (DCOKM) and algorithm (DMOGA). Herein, to support the feasibility of the proposed surrogate model (i.e., DCOKM), the multiobjective and multiconstraint approximation of the carrier roller system MODO is also studied based on the modeling methods of the direct Monte Carlo simulation (MCS), response surface (RS) model, KM, OKM, and the presented DCOKM; Furtherly, to validate the effectiveness of the developed optimal algorithm (i.e., DMOGA), the multiobjective design optimization of the carrier roller system is also performed based on NCGA algorithm, AMGA algorithm, NSGA-II algorithm, and DMOGA algorithm. Note that to ensure the rationality of algorithm comparison, all of optimal algorithms are using DCOKM to approximate the objectives and constraints in optimal algorithm comparisons. The approximation efficacy of different models is listed in Table 4, and the computing efficiency and accuracy of different algorithms are listed in Table 5.

As illustrated in Table 4, the fitting number and fitting time of DCOKM are less than RS, KM, OKM, and the testing time of KM, OKM, and DCOKM are far less than the MCS method. Therefore, the DCOKM holds the highest approximation efficiency. Moreover, the DCOKM is more accurate than RS, KM, and OKM and is almost consistent with the direct MCS. The superior performance of DCOKM is induced by (i) the proposed improved PSO training algorithm can rapidly obtain the optimal parameters of the optimized Kriging model, which improves the nonlinear fitting ability and generalization ability of DCOKM; (ii) the decomposed collaborative sampling strategy in DCOKM brings the distributed parallel computation, which is conducive to reduce nonlinear features and computational task. By integrating the strengths of the optimized Kriging model and decomposed collaborative strategy, DCOKM can efficiently and accurately approximate the multiresponse and multiconstraint. Therefore, the DCOKM is a high-accuracy and high-efficiency surrogate model in multiobjective design optimization.

As revealed in Table 5, the optimization time of the DMOGA algorithm is shorter than NSGA-II, AMGA, and NCGA, and the optimization efficacy of DMOGA is better than NSGA-II, AMGA, and NCGA. The computing advantages of DMOGA is induced by (i) the proposed arithmetic crossover operator can combine nondominated sorting level information, which improves the quality of the offspring population and ensure the diversity of the whole population; (ii) the Poisson mutation operator can generate random numbers in a wider range and overstep the local optimum efficiently, which ensures a faster convergence speed to search for global solutions. Therefore, the DMOGA algorithm is a high-efficiency algorithm for resolving the MODO

Therefore, with the integration of the surrogate model (i.e., DCOKM) and optimal algorithm (i.e., DMOGA), the 
proposed multiobjective design optimization framework is validated to improve optimal efficiency while maintaining acceptable optimal accuracy and thereby is an effective way for the multiobjective optimization design of the multicomponent system.

\section{Conclusions}

The purpose of this study is to develop an efficiently and accurately multiobjective optimization design framework for the multicomponent system. Firstly, to efficiently approximate the multiobjective and multiconstraint, a surrogate model named as the decomposed collaborative optimized Kriging model (DCOKM) is proposed, by absorbing the strengths of the improved PSO algorithm and Kriging model into decomposed collaborative strategy. Then, to accurately solve the multiobjective design optimization model, an optimal algorithm named as the dynamic multiobjective genetic algorithm (DMOGA) is developed, by designing the arithmetic crossover operator and Poisson mutation operator. Regarding the carrier roller system MODO as a study case, the validity and feasibility of multiobjective optimization design framework is proved. Some conclusions are summarized as follows:

(1) From the multiobjective design optimization of the carrier roller system, we discover that the total standard deviation of three carrier rollers is reduced by $92 \%$, and loading distribution is more uniform after optimization

(2) DCOKM has higher accuracy and efficiency than the ordinary Kriging model and optimized Kriging model and is an effective surrogate model for the multiobjective design optimization

(3) The optimal solutions of DMOGA are better than the NSGA-II algorithm due to the superior properties of the arithmetic crossover operator and Poisson mutation operator

(4) The comparison of methods reveals that the proposed multiobjective optimization design framework (i.e., DCOKM and DMOGA) possesses high-efficiency and high-accuracy in multiobjective optimal design of the multicomponent system

\section{Data Availability}

All data generated or analyzed during this study are included in this published article.

\section{Conflicts of Interest}

The authors declare that there are no conflicts of interest regarding the publication of this article.

\section{Acknowledgments}

This paper was cosupported by the National Natural Science Foundation of China (grant nos. 51975028 and 51575024).

\section{References}

[1] N. Bennett, A. Walawalkar, M. Heck, and C. Schindler, "Integration of digging forces in a multi-body-system model of an excavator," Proceedings of the Institution of Mechanical Engineers, Part K: Journal of Multi-Body Dynamics, vol. 230, no. 2, pp. 159-177, 2016.

[2] D. Liao, S. P. Zhu, B. Keshtegar et al., "Probabilistic framework for fatigue life assessment of notched components under size effects," International Journal of Mechanical Sciences, vol. 181, Article ID 105685, 2020.

[3] M. A. Arsic, S. M. Bosnjak, Z. D. Odanovic et al., "Analysis of the spreader track wheels premature damages," Engineering Failure Analysis, vol. 20, pp. 118-136, 2012.

[4] S. P. Zhu, B. Keshtegar, N. T. Trung et al., "Reliability-based structural design optimization: hybridized conjugate mean value approach," Engineering With Computers, vol. 2019, pp. 1-14, 2019.

[5] R. L. Huston and J. W. Kamman, "Testing the accuracy of numerical simulations of multibody systems," Mechanics Research Communications, vol. 28, no. 1, pp. 1-6, 2001.

[6] C. Q. Lm and R. L. Huston, "An energy theorem for developing testing functions for numerical simulations of dynamic systems," Journal of Dynamic Systems, Measurement, and Control, vol. 171, pp. 193-198, 1995.

[7] A. I. J. Forrester and A. J. Keane, "Recent advances in surrogate-based optimization," Progress in Aerospace Sciences, vol. 45, no. 1-3, pp. 50-79, 2009.

[8] D. B. Meng, Y. Li, S. P. Zhu et al., "An enhanced reliability index method and its application in reliability-based collaborative design and optimization," Mathematical Problems in Engineering, vol. 2019, 2019.

[9] S. P. Zhu, B. Keshtegar, S. Chakrabort'y et al., "Novel probabilistic model for searching most probable point in structural reliability analysis," Computer Methods in Applied Mechanics and Engineering, vol. 366, 2020.

[10] K. Salonitis and A. Kolios, "Reliability assessment of cutting tool life based on surrogate approximation methods," The International Journal of Advanced Manufacturing Technology, vol. 71, no. 5-8, pp. 1197-1208, 2014.

[11] K. Song, Y. Zhang, X. Zhuang, X. Yu, and B. Song, "Reliability-based design optimization using adaptive surrogate model and importance sampling-based modified SORA method," Engineering with Computers, vol. 71, 2019.

[12] S.-P. Zhu, H.-Z. Huang, W. Peng, H.-K. Wang, and S. Mahadevan, "Probabilistic physics of failure-based framework for fatigue life prediction of aircraft gas turbine discs under uncertainty," Reliability Engineering \& System Safety, vol. 146, pp. 1-12, 2016.

[13] B. Bai, H. Li, W. Zhang et al., "Application of extremum response surface method-based improved substructure component modal synthesis in mistuned turbine bladed disk," Journal of Sound and Vibration, vol. 2020, Article ID 9596089, 2020.

[14] C.-W. Fei, Y.-S. Choy, D.-Y. Hu, G.-C. Bai, and W.-Z. Tang, "Transient probabilistic analysis for turbine blade-tip radial clearance with multi-component and multi-physics fields based on DCERSM," Aerospace Science and Technology, vol. 50, pp. 62-70, 2016.

[15] L. K. Song, G. C. Bai, and C. W. Fei, "Dynamic surrogate modeling approach for probabilistic creep-fatigue life evaluation of turbine disks," Aerospace Science and Technology, vol. 95, 2019. 
[16] C. Lu, Y.-W. Feng, C.-W. Fei, and S.-Q. Bu, "Improved decomposed-coordinated kriging modeling strategy for dynamic probabilistic analysis of multicomponent structures," IEEE Transactions on Reliability, vol. 69, no. 2, pp. 440-457, 2020.

[17] Y. Huang, G. C. Bai, L. K. Song et al., "Distributed collaborative modeling approach for probabilistic fatigue life evaluation of turbine rotor," Materials, vol. 13, 2020.

[18] L. K. Song, C. W. Fei, and G. C. Bai, "Dynamic neural network method-based improved PSO and BR algorithms for transient probabilistic analysis of flexible mechanism," Advanced Engineering Informatics, vol. 33, pp. 144-153, 2019.

[19] L.-K. Song, G.-C. Bai, C.-W. Fei, and R. P. Liem, "Transient probabilistic design of flexible multibody system using a dynamic fuzzy neural network method with distributed collaborative strategy," Proceedings of the Institution of $\mathrm{Me}$ chanical Engineers, Part G: Journal of Aerospace Engineering, vol. 233, no. 11, pp. 4077-4090, 2019.

[20] L.-K. Song and G.-C. Bai, "Multi-surrogate collaboration approach for creep-fatigue reliability assessment of turbine rotor," IEEE Access, vol. 8, pp. 39861-39874, 2020.

[21] V. Cherkassky and Y. Ma, "Practical selection of SVM parameters and noise estimation for SVM regression," Neural Networks, vol. 17, no. 1, pp. 113-126, 2004.

[22] B. Bai, Z. Li, J. Zhang, and W. Zhang, "Application of support vector machine-based classification extremum method in flexible mechanism," Journal of Mechanisms and Robotics, vol. 12 , no. 4, 2020.

[23] C. Jin and S.-W. Jin, "Software reliability prediction model based on support vector regression with improved estimation of distribution algorithms," Applied Soft Computing, vol. 15, pp. 113-120, 2014.

[24] M. Li, "An improved kriging-assisted multi-objective genetic algorithm," Journal of Mechanical Design, vol. 133, no. 7, 2011.

[25] M. Li, G. Li, and S. Azarm, "A kriging metamodel assisted multi-objective genetic algorithm for design optimization," Journal of Mechanical Design, vol. 130, no. 3, 2008.

[26] I. Kaymaz, "Application of Kriging method to structural reliability problems," Structural Safety, vol. 27, no. 2, pp. 133-151, 2005.

[27] L.-K. Song, J. Wen, C.-W. Fei, and G.-C. Bai, "Distributed collaborative probabilistic design of multi-failure structure with fluid-structure interaction using fuzzy neural network of regression," Mechanical Systems and Signal Processing, vol. 104, pp. 72-86, 2018.

[28] L.-K. Song, G.-C. Bai, and C.-W. Fei, "Probabilistic LCF life assessment for turbine discs with DC strategy-based wavelet neural network regression," International Journal of Fatigue, vol. 119, pp. 204-219, 2019.

[29] L.-K. Song, G.-C. Bai, and C.-W. Fei, "Multi-failure probabilistic design for turbine bladed disks using neural network regression with distributed collaborative strategy," Aerospace Science and Technology, vol. 92, pp. 464-477, 2019.

[30] L.-K. Song, C.-W. Fei, J. Wen, and G.-C. Bai, "Multi-objective reliability-based design optimization approach of complex structure with multi-failure modes," Aerospace Science and Technology, vol. 64, pp. 52-62, 2017.

[31] W. Wang, S. Yuan, J. Pei, and J. Zhang, "Optimization of the diffuser in a centrifugal pump by combining response surface method with multi-island genetic algorithm," Proceedings of the Institution of Mechanical Engineers, Part E: Journal of Process Mechanical Engineering, vol. 231, no. 2, pp. 191-201, 2017.
[32] J. Liu, G. Yu, Y. Li, H. Wang, and W. Xiao, "Multidisciplinary design optimization of crankshaft structure based on cooptimization and multi-island genetic algorithm," Mathematical Problems in Engineering, vol. 2016, Article ID 9596089, 11 pages, 2016.

[33] J. Huang, L. Zheng, C. K. Mechefske, and B. Han, "Optimization design and experimental study of a two-disk rotor system based on multi-island Genetic Algorithm," International Journal of Turbo \& Jet-Engines, vol. 36, no. 1, pp. 1-8, 2019.

[34] L. Wang and C. S. Zhao, "System optimal design based on neighborhood cultivation genetic algorithm combined with modified method of feasible directions," Advanced Materials Research, vol. 143-144, pp. 980-983, 2010.

[35] Z. Su, F. Xu, L. Hua, H. Chen, K. Wu, and S. Zhang, "Design optimization of minivan MacPherson-strut suspension system based on weighting combination method and neighborhood cultivation genetic algorithm," Proceedings of the Institution of Mechanical Engineers, Part D: Journal of Automobile Engineering, vol. 233, no. 3, pp. 650-660, 2019.

[36] K. Deb, A. Pratap, S. Agarwal, and T. Meyarivan, "A fast and elitist multiobjective genetic algorithm: nsga-II," IEEE Transactions on Evolutionary Computation, vol. 6, no. 2, pp. 182-197, 2002.

[37] J. Liu and X. Chen, "An improved NSGA-II algorithm based on crowding distance elimination strategy," International Journal of Computational Intelligence Systems, vol. 12, no. 2, pp. 513-518, 2019.

[38] Z. Zhao, B. Liu, C. Zhang, and H. Liu, "An improved adaptive NSGA-II with multi-population algorithm," Applied Intelligence, vol. 49, no. 2, pp. 569-580, 2019.

[39] S. Tiwari, G. Fadel, and K. Deb, "AMGA2: improving the performance of the archive-based micro-genetic algorithm for multi-objective optimization," Optimization and Engineering, vol. 43, no. 4, pp. 371-401, 2011.

[40] S. Ahn, N. Kim, S. Lee et al., "Performance analysis and optimization of AMGA for the large-scale virtual screening," Software: Practice and Experience, vol. 39, no. 12, pp. 10551072, 2009.

[41] K. Deb and R. B. Agrawal, "Simulated binary crossover for continuous search space," Complex Systems, vol. 9, no. 3, pp. 115-148, 2000.

[42] J.-H. Yi, L.-N. Xing, G.-G. Wang et al., "Behavior of crossover operators in NSGA-III for large-scale optimization problems," Information Sciences, vol. 509, pp. 470-487, 2020.

[43] J. Sacks, S. B. Schiller, and W. J. Welch, "Designs for computer experiments," Technometrics, vol. 31, no. 1, pp. 41-47, 1989.

[44] L. Tang, H. Wang, G. Li, and F. Xu, "Adaptive heuristic search algorithm for discrete variables based multi-objective optimization," Structural and Multidisciplinary Optimization, vol. 48, no. 4, pp. 821-836, 2013.

[45] X. Yao, X. Guo, Y. Feng, and C. Yu, "Research on the uprising dynamics of a double-carriage overhead crane system," Proceedings of the Institution of Mechanical Engineers, Part C: Journal of Mechanical Engineering Science, vol. 225, no. 1, pp. 112-122, 2011.

[46] C. Schmitke, K. Morency, and J. McPhee, "Using graph theory and symbolic computing to generate efficient models for multi-body vehicle dynamics," Proceedings of the Institution of Mechanical Engineers, Part K: Journal of Multi-Body Dynamics, vol. 222, no. 4, pp. 339-352, 2008. 